Supplement of Biogeosciences, 17, 917-943, 2020

https://doi.org/10.5194/bg-17-917-2020-supplement

(C) Author(s) 2020. This work is distributed under

the Creative Commons Attribution 4.0 License.

(c) (1)

Supplement of

\title{
Dissolved iron in the North Atlantic Ocean and Labrador Sea along the GEOVIDE section (GEOTRACES section GA01)
}

Manon Tonnard et al.

Correspondence to: Géraldine Sarthou (geraldine.sarthou@univ-brest.fr) and Hélène Planquette (helene.planquette@univbrest.fr)

The copyright of individual parts of the supplement might differ from the CC BY 4.0 License. 
Figure S1: Section plot of Total Chlorophyll-a (TChl-a) concentrations $\left(\mathrm{mg} \mathrm{m}^{-3}\right)$ measured for the GA01 voyage. The black contour lines highlight the TChl- $a$ concentrations and the white contour lines highlight the dissolved iron (DFe) concentrations. The red dashed line indicates the depth of the Surface Mixed Layer (SML) (see text for details). (Ocean Data View (ODV) software, version 4.7.6, R. Schlitzer, http://odv.awi.de, 2016, last( access: Jan 30 2020).

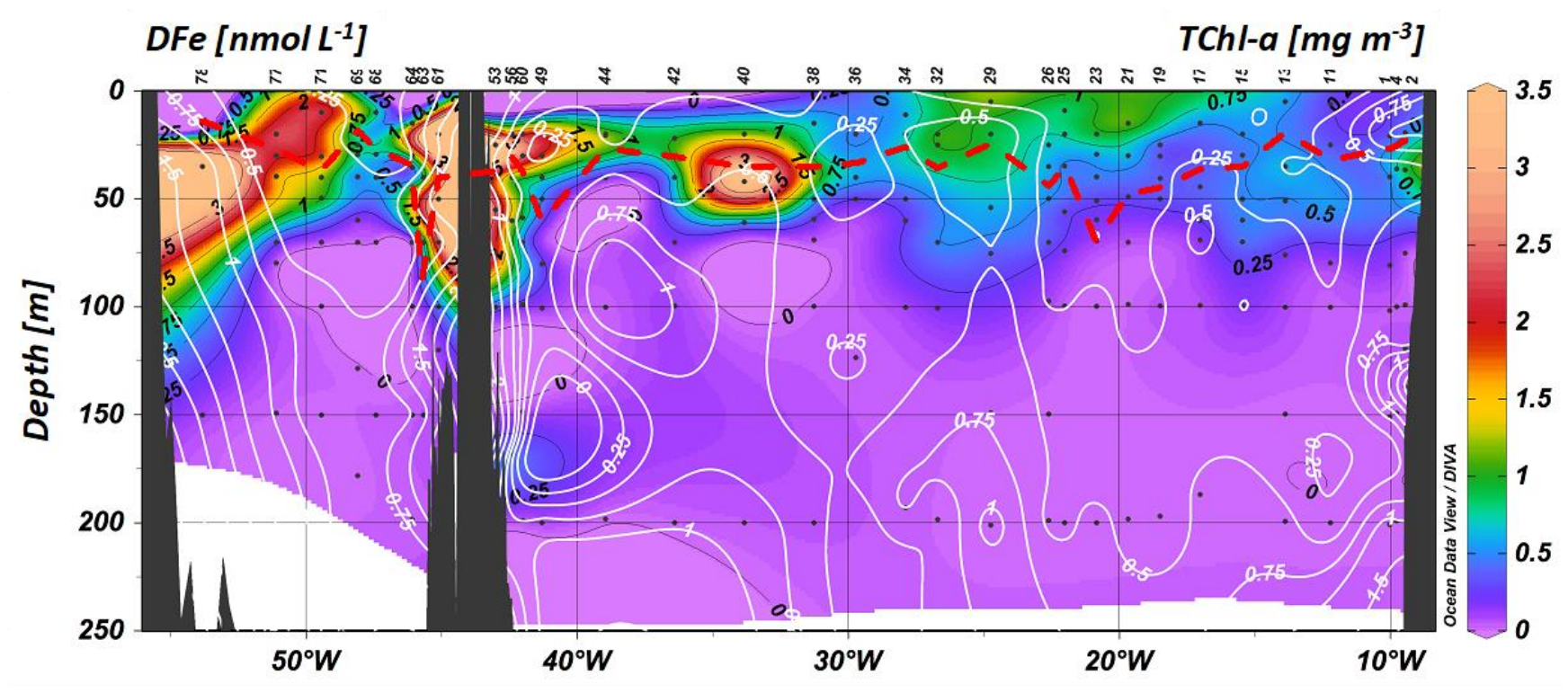


Figure S2: Mean profiles of dissolved iron (Fe) along the North Atlantic section in the West European Basin (purple), Iceland Basin (blue), Irminger Sea (green) and Labrador Sea (red) over the depth intervals: 0-100 m, 100-250 m, 250-500 m, 500-1000 m, 1000$1500 \mathrm{~m}, 1500-2000 \mathrm{~m}, 2000-3000 \mathrm{~m}, 3000-4000 \mathrm{~m}, 4000-5500 \mathrm{~m}$ without considering stations located above the continental plateau.

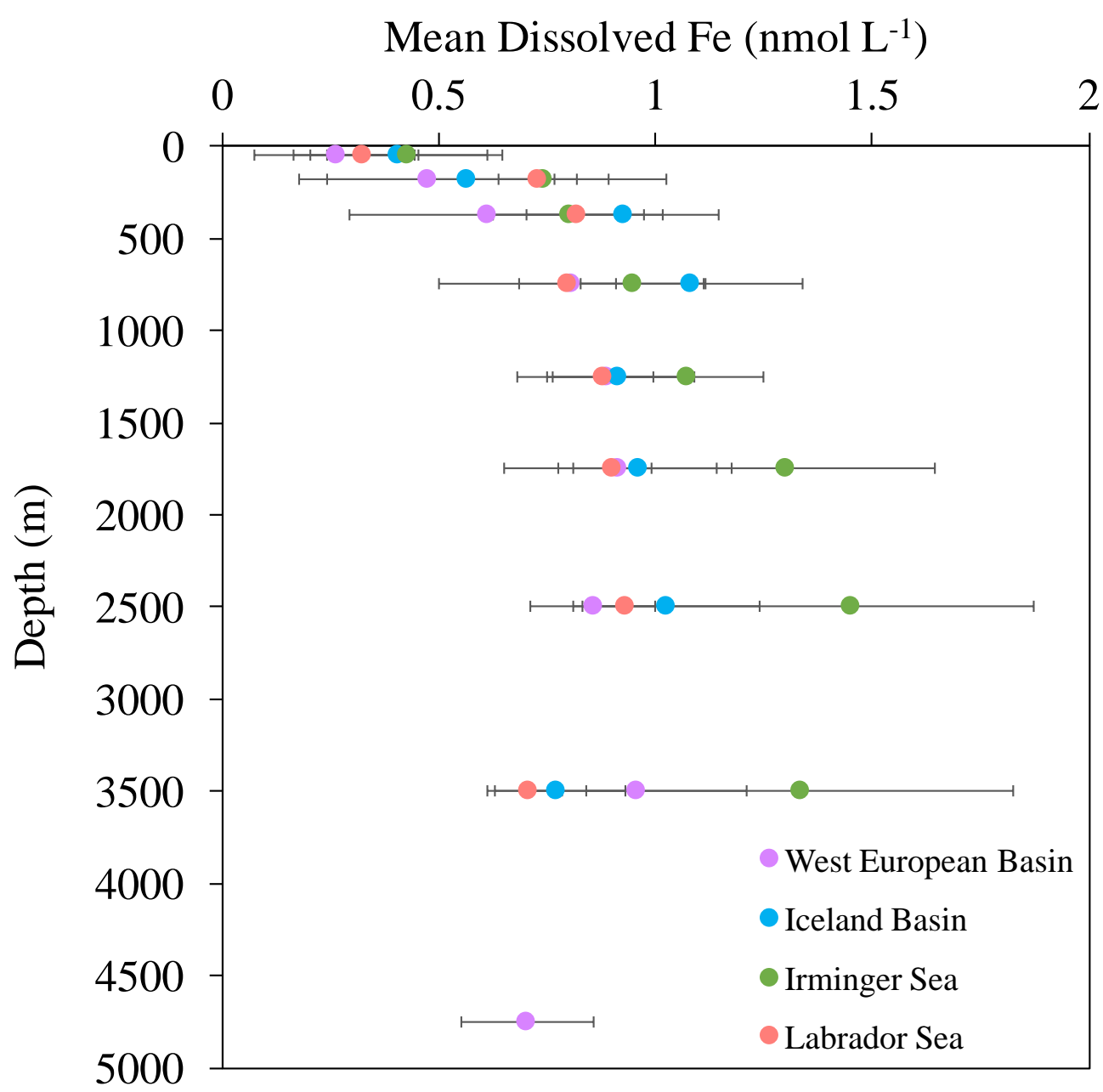


Figure S3: Box and whisker plot of dissolved iron (DFe) in nmol $\mathrm{L}^{-1}$ per water mass and basin. Color coding representing from West to East: the Labrador Sea (red), the Irminger Sea (green), the Iceland Basin (blue) and the West European Basin (purple). Note that stations 1 and 17 were not considered in this plot. SAIW: Sub-Arctic Intermediate Water, ENACW: East North Atlantic Central Water, IrSPMW: Irminger Sub-Polar Mode Water, IcSPMW: Iceland Sub-Polar Mode Water, MOW: Mediterranean Overflow

5 Water, LSW: Labrador Sea Water, ISOW: Iceland-Scotland Overflow Water, DSOW: Denmark Strait Overflow Water, NEADW: North East Atlantic Deep Water. The red dotted line represents the DFe median value $\left(0.77 \mathrm{nmol} \mathrm{L}^{-1}\right)$.

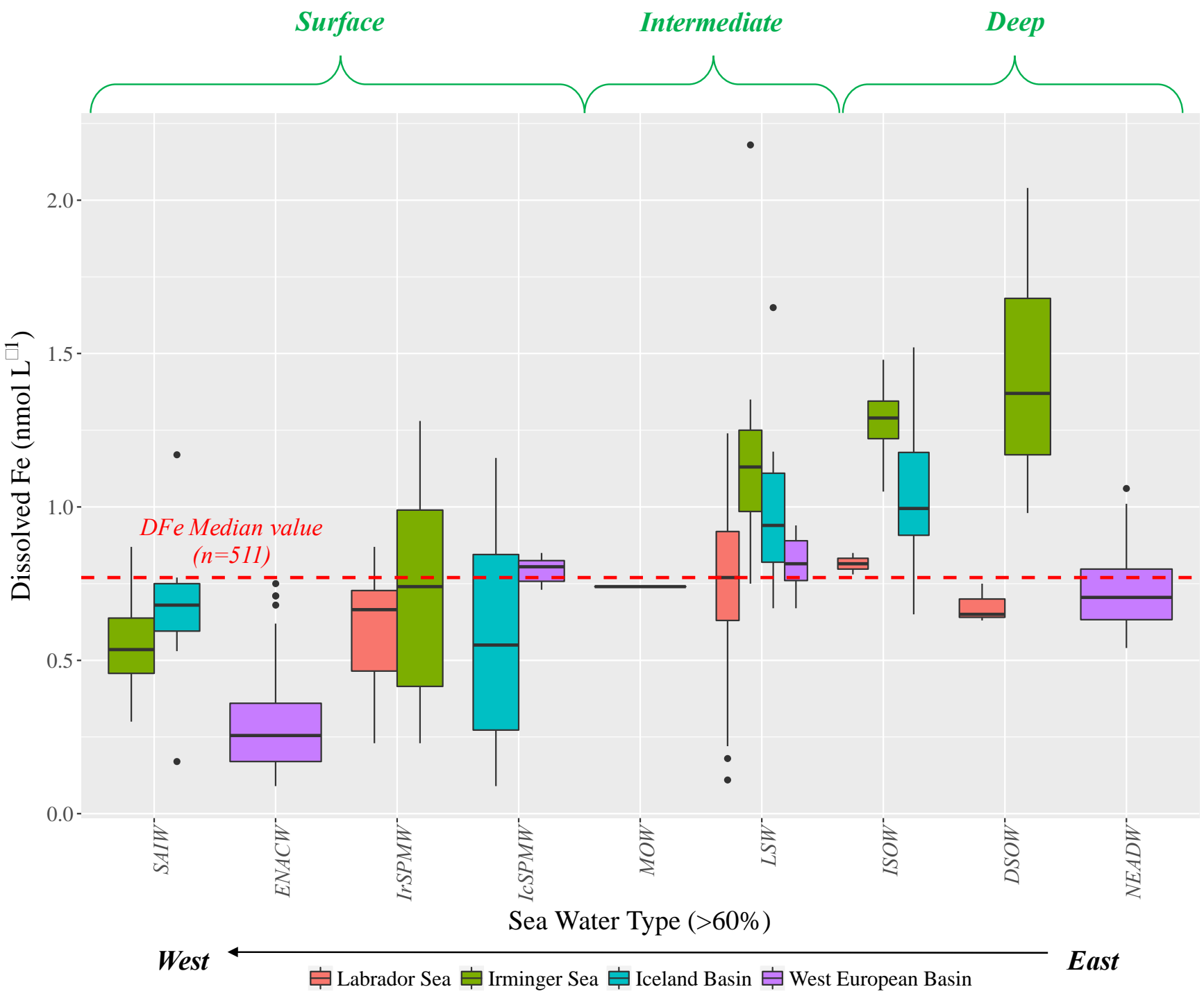


Figure S4: Surface layer of DFe concentrations, new measurements are shown in red dots (GEOVIDE voyage), while previous studies are displayed in black (Achterberg et al., 2018; Bergquist et al., 2007; Blain et al., 2004; Boye et al., 2006, 2003; de Jong et al., 2007; Gledhill et al., 1998; Hatta et al., 2015; Klunder et al., 2012; Laës et al., 2003; Martin et al., 1993; Measures et al., 2008; Mills et al., 2008; Mohamed et al., 2011; Nédélec et al., 2007; Nielsdóttir et al., 2009; Pohl et al., 2011; Rijkenberg et al., 2014; Sarthou et al., 5 2007, 2003; Sedwick et al., 2005; Ussher et al., 2013; Witter and Luther III, 1998; Wu and Boyle, 2002; Wu and Luther III, 1996, 1994; Wu et al., 2001).

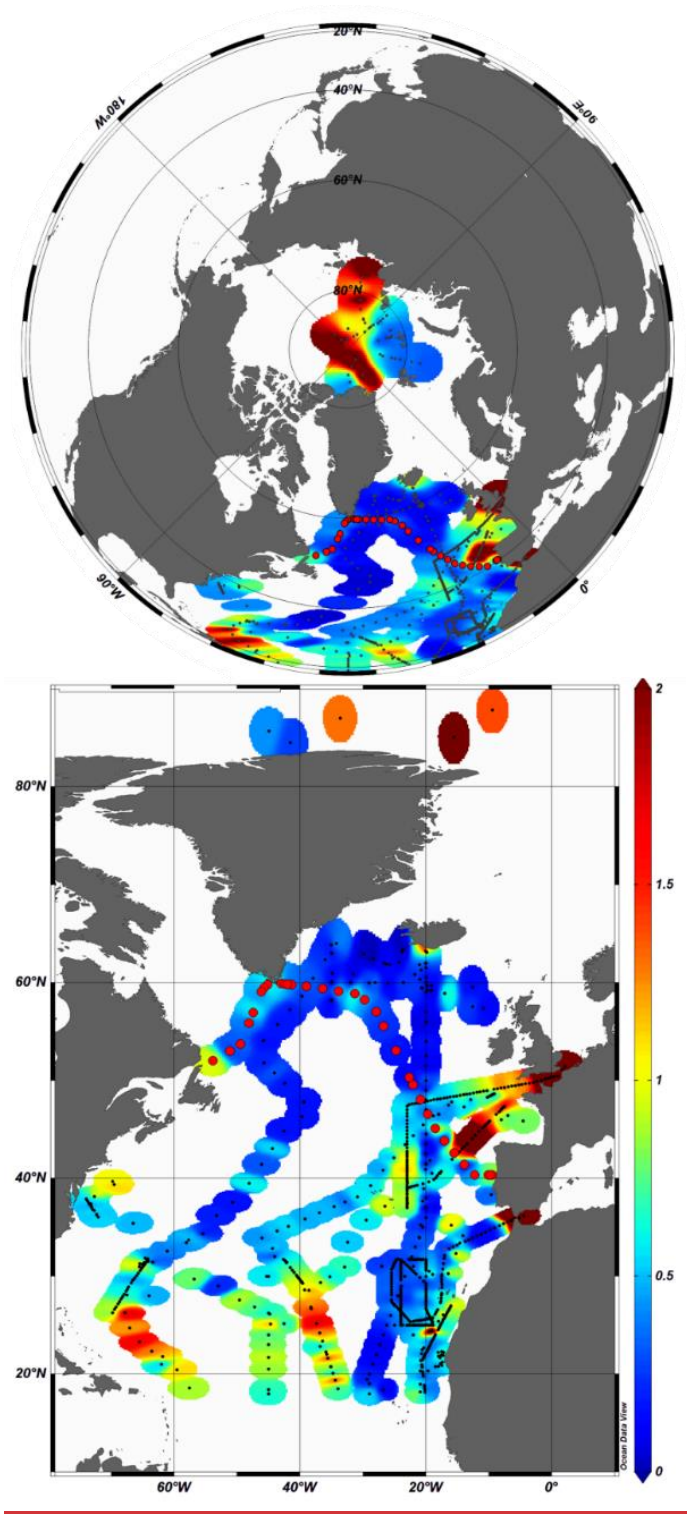


Figure S5: A) Plot of dissolved iron (DFe) concentrations as a function of the percentage of Polar Intermediate Water (PIW) contribution for open-ocean stations (stations 44, 49, 60, 63, 68, 69, 71 and 77). Station 44 highlighted in green and dashed-line representing the linear regression line between DFe concentrations and percentage of PIW contribution for all stations except station 44. B) Plot of dissolved (DFe, black dots) and particulate iron (PFe, open dots, Gourain et al., in prep.) for station 44 (from $2220 \mathrm{~m}$ 5 depth to the bottom) as a function of the percentage of mixing between Iceland-Scotland Overflow Water (ISOW) as opposed to Polar Intermediate Water (PIW) and Denmark Strait Overflow Water (DSOW) (Garcia-Ibanez et al., 2015) with polynomial (DFe) and exponential (PFe) regression equations.

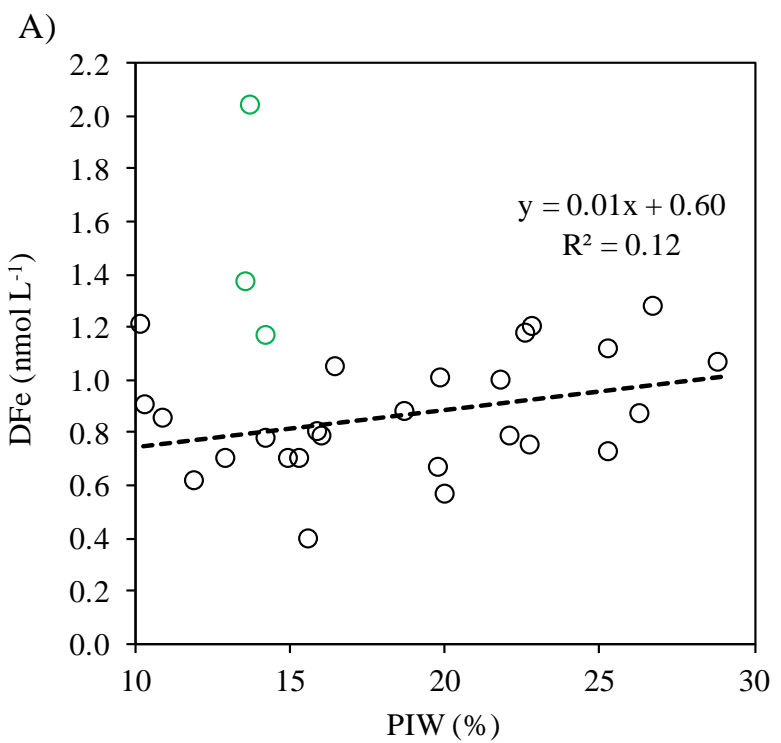

B)

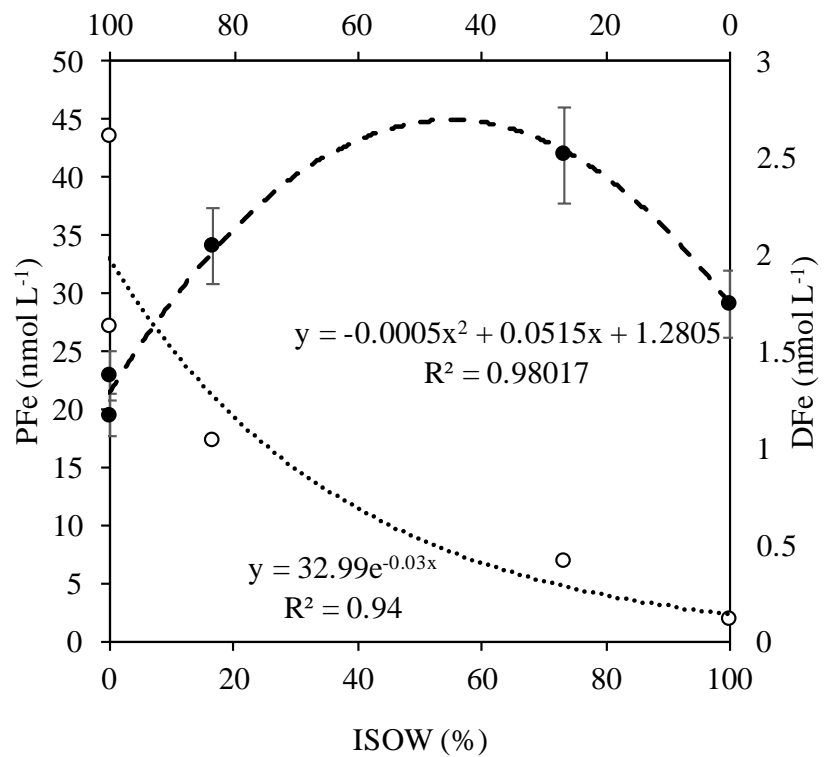


Figure S6: Plots of the first two dimensions of a Principal Component Analysis (PCA) performed on A) the following variables: Apparent Oxygen Utilization (AOU), dissolved aluminium (DAl, Menzel Barraqueta et al., 2018), particulate iron, aluminum and manganese oxides (PFe, PAl and MnO , Gourain et al., in prep.) and B) for samples which presented a transmissometry lower than $99 \%$ and below 500 m depth to avoid surface processes. Note that the color coding corresponds to different water masses (contribution $>60 \%$ of the whole water mass pool) with the Denmark Strait Overflow Water 5 (DSOW) in grey, the East North Atlantic Central Water (ENACW) in yellow, the Irminger Sub-Polar Mode Water (IrSPMW) in blue, the IcelandScotland Overflow Water (ISOW) in green, the Labrador Sea Water (LSW) in red, the Mediterranean Overflow Water (MOW) in orange, the North East Atlantic Deep Water (NEADW) in pink and mixing of multiple water masses (NA) in white. Plots of dissolved iron (DFe) plotted as a function of distance height above the seafloor for $\mathrm{C}$ ) the first dimension of the PCA and D) the second dimension of the PCA. Note that positive and negative values are represented in blue and red, respectively and that dot size are function of the particulate iron and manganese oxide ratios $\left(\mathrm{PFe}: \mathrm{MnO}_{2}, \mathrm{~mol}^{\mathrm{mol}}{ }^{-1}\right)$.

A)

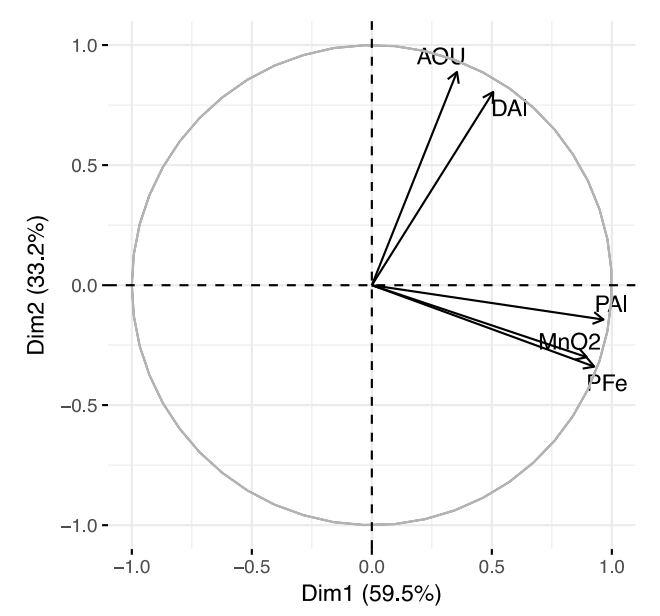

C)

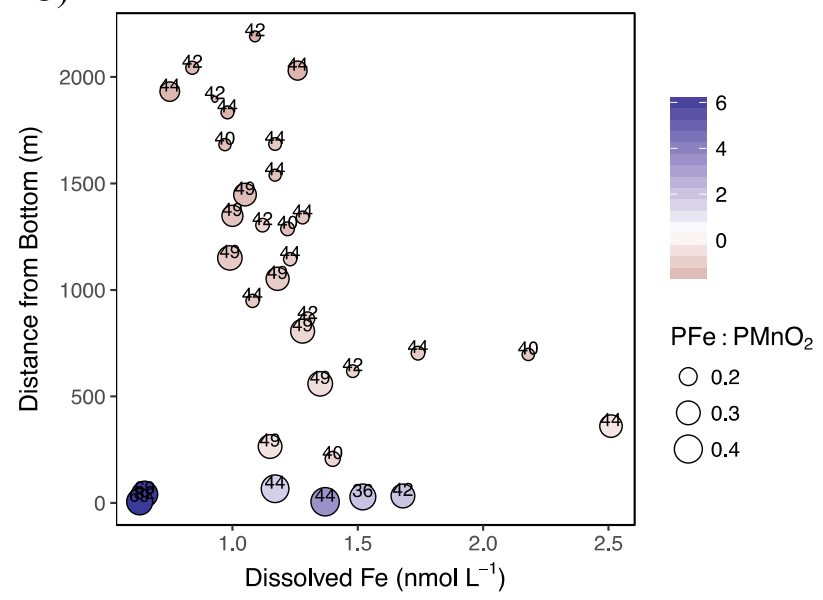

B)

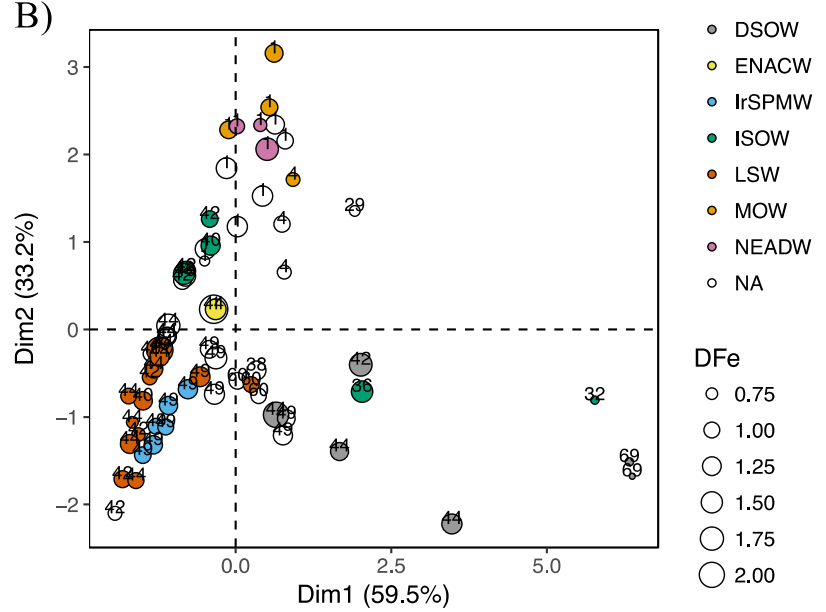

D)

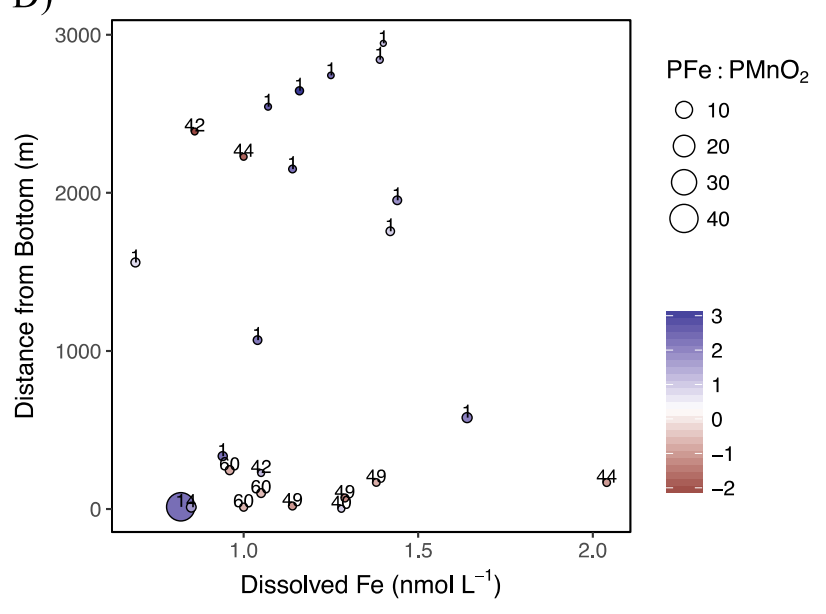


Figure S7: Vertical profiles of the DFe:NO3 ratio over the upper $200 \mathrm{~m}$ of the water column along the GEOVIDE section. Profiles from the West European Basin are plotted in black, from the Iceland Basin in grey, from the Irminger Sea in green and from the Labrador Sea in red. Stations located above the continental Plateau (stations 1, 2 and 4 from the Iberian Margin; stations 53 and 61

5 from the Greenland shelf; station 78 from the Newfoundland Margin) are represented with dotted lines. The vertical dashed lines (light blue) indicate lower and upper limits 5 of phytoplankton cellular DFe:NO3 ratios under Fe replete conditions Ho et al., 2003; Sunda and Huntsman, 1995; Twining et al., 2004.

\section{Fe deficient $\quad$ Fe replete}

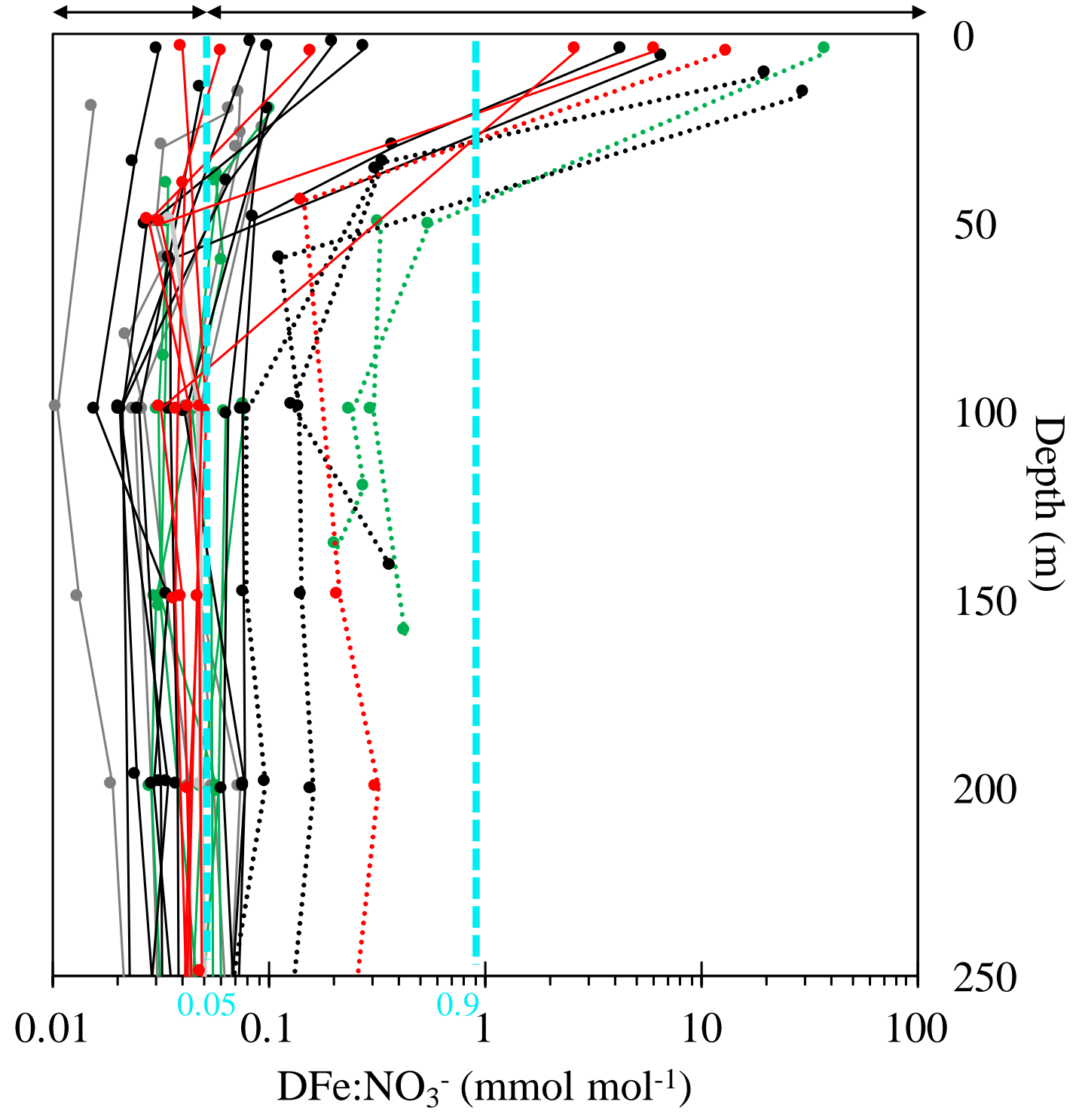


Figure S8: (A) Apparent Oxygen Utilization (AOU) vs. $\mathrm{NO}_{3}$, (B) AOU vs. DFe, and (C) DFe vs. $\mathrm{NO}_{3}$ in the subpolar gyre between $50 \mathrm{~m}$ and $250 \mathrm{~m}$ at station $(29-49,56,60,63-77)$.

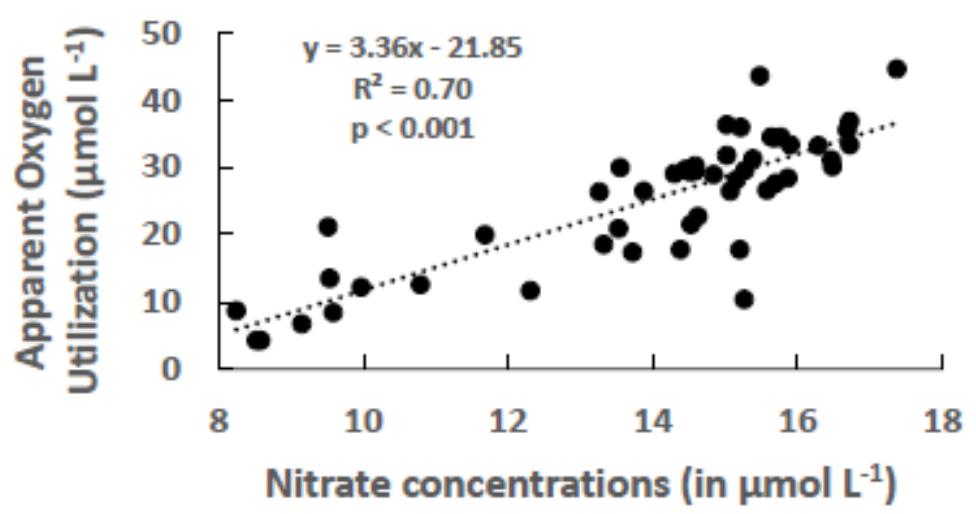

(A)

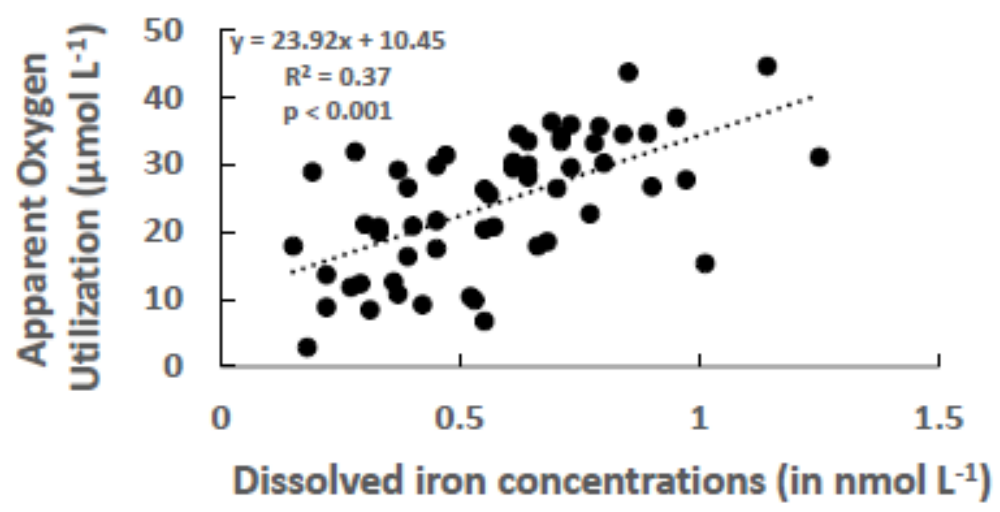

(B)

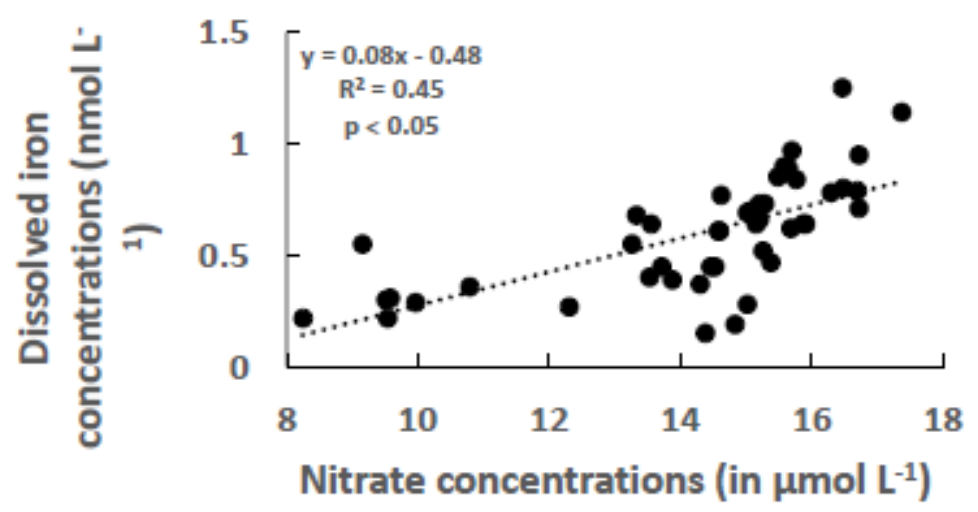

(C) 
Figure S9: Surface map of the DFe:NO3 ratios along the GEOVIDE section.

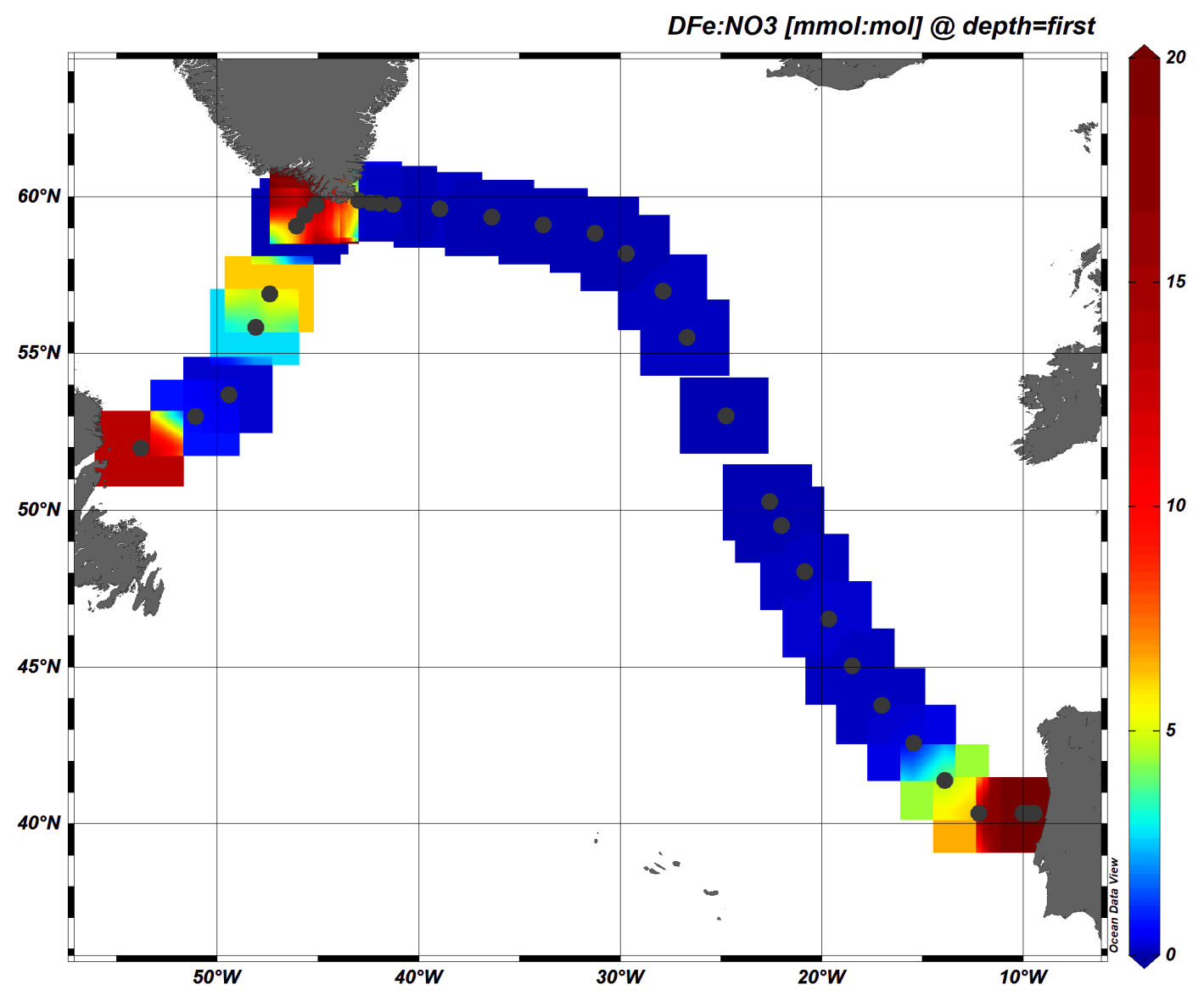


Figure S10: Section plot of the Fe* tracer in the North Atlantic Ocean with a remineralization rate $\left(R_{\mathrm{Fe}: \mathrm{N})}\right)$ of $0.05 \mathrm{mmol} \mathrm{mol}^{-1}$ from $100 \mathrm{~m} \mathrm{depth}$ to bottom waters. A contour line of $\mathrm{O}$ separates areas of negative $\mathrm{Fe}^{*}$ from areas with positive $\mathrm{Fe}^{*}$. Positive values of $\mathrm{Fe}^{*}$ imply there is enough iron to support complete consumption of $\mathrm{NO}_{3}{ }^{-}$when this water is brought to surface, and negative $\mathrm{Fe}^{*}$ imply a deficit. See text for details.

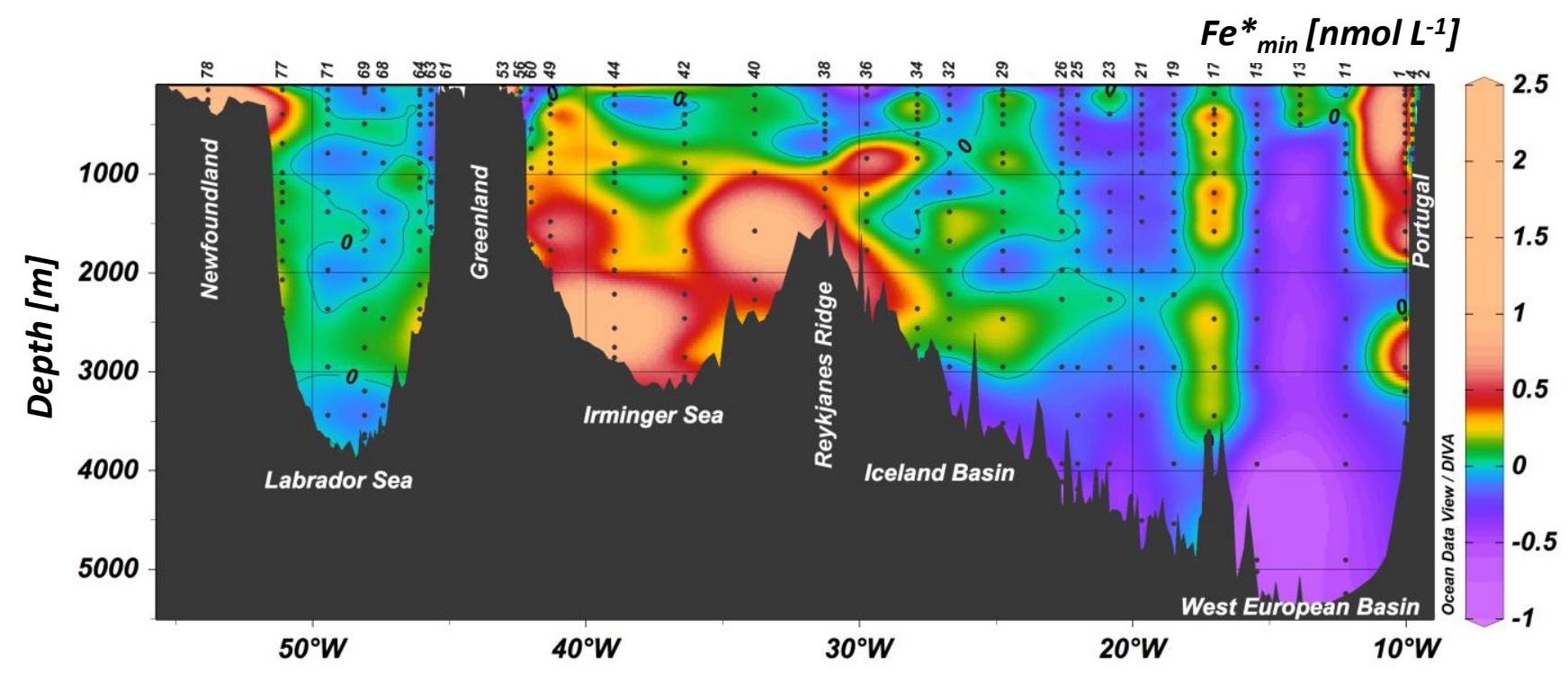


Figure S11: Box and whisker plot of $\mathrm{Fe}^{*}$ in units of $\mathrm{nmol} \mathrm{L}^{-1}$ as determined per water mass and basin with a Fe: $\mathrm{N}$ uptake rate of 0.05 . Color coding representing from West to East, the Labrador Sea (red), the Irminger Sea (green), the Iceland Basin (blue) and the West European Basin (purple). Abbreviation referring to SAIW: Sub-Arctic Intermediate Water, ENACW: East North Atlantic Central Water, IrSPMW: Irminger Sub-Polar Mode Water, IcSPMW: Iceland Sub-Polar Mode Water, MOW: Mediterranean Overflow Water, LSW: Labrador Sea Water, ISOW: Iceland-Scotland 5 Overflow Water, DSOW: Denmark Strait Overflow Water, NEADW: North East Atlantic Deep Water.

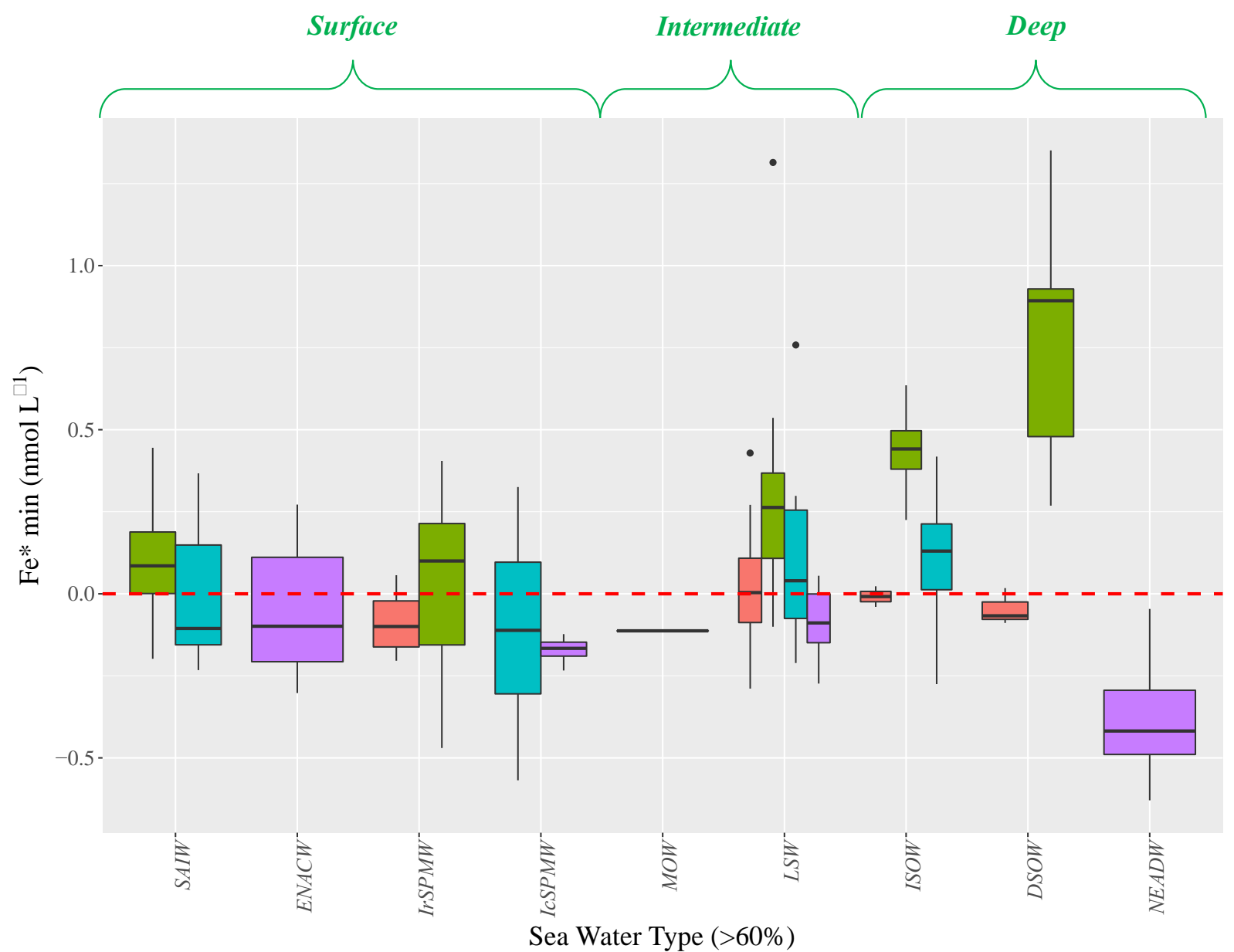

West

官Labrador Sea 追 Irminger Sea 白 Iceland Basin 追 West European Basin 
Table S1: Table of dissolved iron (DFe) data for the whole GEOVIDE section. Bold characters denote stations located above the shelves. Note that $\mathrm{QC}$ refers to the data quality $(1=\operatorname{good}$ data, $2=$ questionable, $3=$ bad data, were removed $)$.

\begin{tabular}{|c|c|c|c|c|c|c|c|c|c|}
\hline $\begin{array}{c}\text { Station } \\
(\#)\end{array}$ & $\begin{array}{l}\text { Lat } \\
\left({ }^{\circ} \mathbf{N}\right) \\
\end{array}$ & $\begin{array}{l}\text { Lon } \\
\left({ }^{\circ} \mathbf{E}\right) \\
\end{array}$ & $\begin{array}{c}\text { Bottle } \\
(\#)\end{array}$ & $\begin{array}{c}\text { Depth } \\
\text { (m) }\end{array}$ & (nmol kg-1) & $\begin{array}{c}\text { DFe } \\
(\mathrm{nmol} \mathrm{L-1)}\end{array}$ & & SD & $\begin{array}{l}\text { QC } \\
(\#)\end{array}$ \\
\hline \multirow[t]{23}{*}{1} & 40.333 & -10.036 & 24 & 21 & 1.04 & 1.07 & \pm & 0.12 & 2 \\
\hline & & & 22 & 60 & 0.50 & 0.51 & \pm & 0.06 & 1 \\
\hline & & & 21 & 81 & 0.72 & 0.74 & \pm & 0.09 & 1 \\
\hline & & & 20 & 102 & 0.82 & 0.84 & \pm & 0.10 & 1 \\
\hline & & & 19 & 151 & 0.94 & 0.96 & \pm & 0.11 & 1 \\
\hline & & & 18 & 201 & 1.23 & 1.26 & \pm & 0.15 & 2 \\
\hline & & & 17 & 300 & 1.05 & 1.08 & \pm & 0.13 & 1 \\
\hline & & & 16 & 387 & 1.28 & 1.31 & \pm & 0.15 & 1 \\
\hline & & & 15 & 501 & 1.40 & 1.44 & \pm & 0.17 & 1 \\
\hline & & & 14 & 589 & 1.37 & 1.40 & \pm & 0.16 & 1 \\
\hline & & & 13 & 693 & 1.36 & 1.39 & \pm & 0.16 & 1 \\
\hline & & & 12 & 792 & 1.22 & 1.25 & \pm & 0.15 & 1 \\
\hline & & & 11 & 889 & 1.13 & 1.16 & \pm & 0.14 & 1 \\
\hline & & & 10 & 990 & 1.04 & 1.07 & \pm & 0.12 & 1 \\
\hline & & & 9 & 1185 & 1.18 & 1.21 & \pm & 0.14 & 1 \\
\hline & & & 8 & 1384 & 1.11 & 1.14 & \pm & 0.13 & 1 \\
\hline & & & 7 & 1582 & 1.40 & 1.44 & \pm & 0.17 & 2 \\
\hline & & & 6 & 1778 & 1.39 & 1.42 & \pm & 0.17 & 2 \\
\hline & & & 5 & 1976 & 0.67 & 0.69 & \pm & 0.08 & 1 \\
\hline & & & 4 & 2466 & 1.01 & 1.04 & \pm & 0.12 & 1 \\
\hline & & & 3 & 2957 & 1.60 & 1.64 & \pm & 0.19 & 2 \\
\hline & & & 2 & 3201 & 0.92 & 0.94 & \pm & 0.11 & 1 \\
\hline & & & 1 & 3521 & 0.80 & 0.82 & \pm & 0.10 & 1 \\
\hline \multirow[t]{7}{*}{2} & 40.333 & -9.46 & 21 & 19 & 0.99 & 1.01 & \pm & 0.04 & 1 \\
\hline & & & 19 & 37 & 0.36 & 0.37 & \pm & 0.01 & 1 \\
\hline & & & 14 & 50 & 0.46 & 0.47 & \pm & 0.02 & 1 \\
\hline & & & 13 & 75 & 0.61 & 0.63 & \pm & 0.02 & 1 \\
\hline & & & 9 & 99 & 0.73 & 0.75 & \pm & 0.03 & 1 \\
\hline & & & 5 & 119 & 0.56 & 0.57 & \pm & 0.02 & 1 \\
\hline & & & 1 & 137 & 2.97 & 3.04 & \pm & 0.12 & 1 \\
\hline 4 & 40.333 & -9.767 & 23 & 21 & 0.71 & 0.73 & \pm & 0.03 & 2 \\
\hline
\end{tabular}




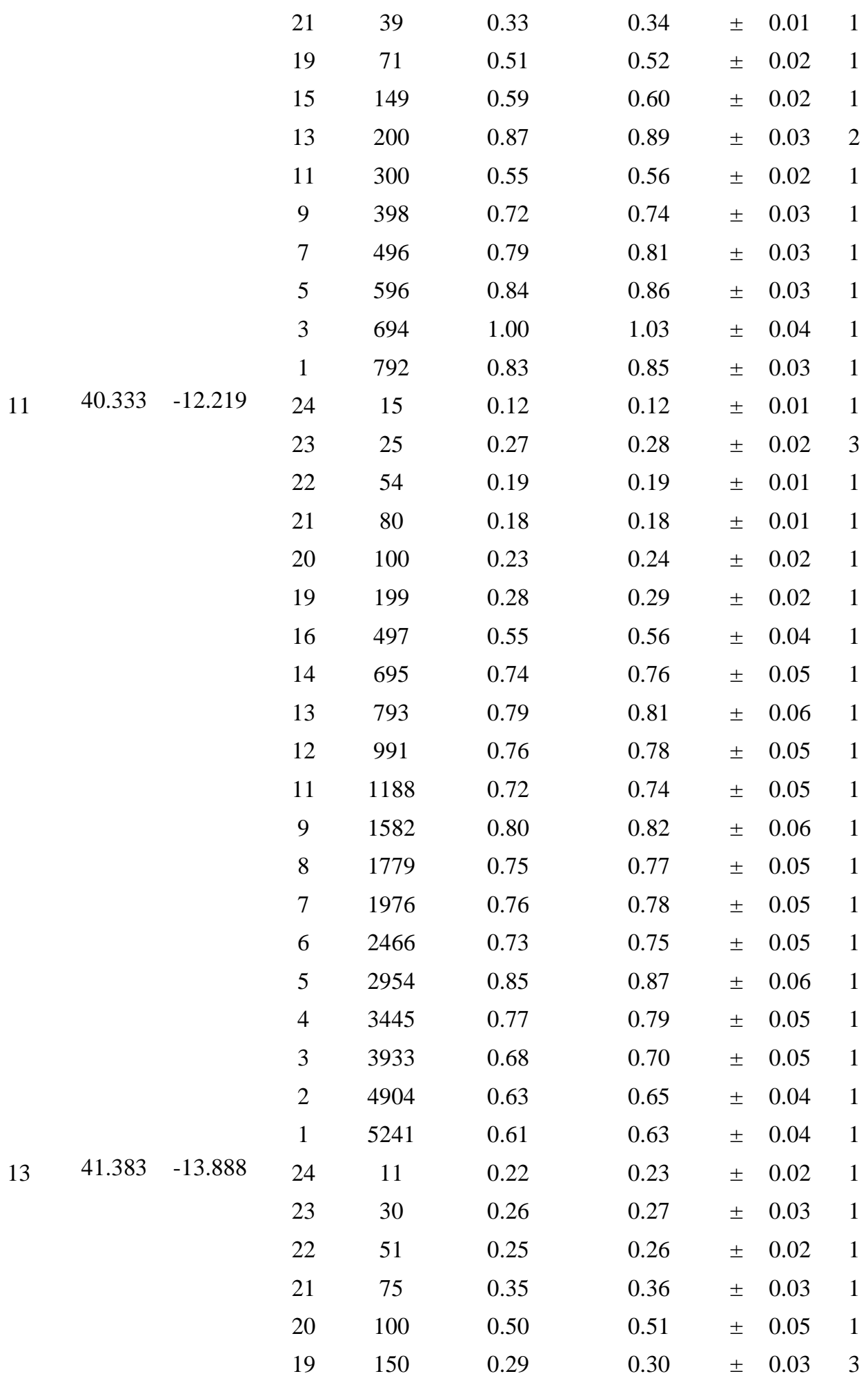




\begin{tabular}{|c|c|c|c|c|c|c|c|c|}
\hline & & & 16 & 199 & 0.59 & 0.60 & \pm & 0.06 \\
\hline & & & 15 & 298 & 0.60 & 0.62 & \pm & 0.06 \\
\hline & & & 14 & 397 & 0.69 & 0.71 & \pm & 0.07 \\
\hline & & & 13 & 496 & 0.69 & 0.71 & \pm & 0.07 \\
\hline \multirow[t]{22}{*}{15} & 42.581 & -15.461 & 24 & 20 & 0.24 & 0.25 & \pm & 0.02 \\
\hline & & & 23 & 30 & 0.19 & 0.19 & \pm & 0.02 \\
\hline & & & 22 & 49 & 0.14 & 0.14 & \pm & 0.01 \\
\hline & & & 21 & 60 & 0.32 & 0.33 & \pm & 0.03 \\
\hline & & & 20 & 70 & 0.25 & 0.26 & \pm & 0.02 \\
\hline & & & 19 & 99 & 0.17 & 0.17 & \pm & 0.02 \\
\hline & & & 16 & 298 & 0.26 & 0.27 & \pm & 0.03 \\
\hline & & & 15 & 397 & 0.34 & 0.35 & \pm & 0.03 \\
\hline & & & 14 & 496 & 0.44 & 0.45 & \pm & 0.04 \\
\hline & & & 13 & 644 & 0.65 & 0.67 & \pm & 0.07 \\
\hline & & & 12 & 793 & 0.65 & 0.67 & \pm & 0.06 \\
\hline & & & 11 & 989 & 0.74 & 0.76 & \pm & 0.07 \\
\hline & & & 10 & 1089 & 0.72 & 0.74 & \pm & 0.07 \\
\hline & & & 9 & 1384 & 0.60 & 0.62 & \pm & 0.06 \\
\hline & & & 8 & 1581 & 0.68 & 0.70 & \pm & 0.07 \\
\hline & & & 7 & 1779 & 0.70 & 0.72 & \pm & 0.07 \\
\hline & & & 6 & 1975 & 0.65 & 0.67 & \pm & 0.06 \\
\hline & & & 5 & 2466 & 0.71 & 0.73 & \pm & 0.07 \\
\hline & & & 4 & 2956 & 0.73 & 0.75 & \pm & 0.07 \\
\hline & & & 3 & 3932 & 0.56 & 0.57 & \pm & 0.06 \\
\hline & & & 2 & 4904 & 0.53 & 0.54 & \pm & 0.05 \\
\hline & & & 1 & 5020 & 0.57 & 0.58 & \pm & 0.06 \\
\hline \multirow[t]{10}{*}{17} & 43.78 & -17.032 & 24 & 15 & 0.17 & 0.17 & \pm & 0.01 \\
\hline & & & 23 & 30 & 0.88 & 0.91 & \pm & 0.06 \\
\hline & & & 22 & 44 & 0.52 & 0.53 & \pm & 0.04 \\
\hline & & & 21 & 60 & 0.54 & 0.55 & \pm & 0.04 \\
\hline & & & 20 & 69 & 0.56 & 0.57 & \pm & 0.04 \\
\hline & & & 19 & 99 & 0.54 & 0.55 & \pm & 0.04 \\
\hline & & & 16 & 199 & 0.56 & 0.57 & \pm & 0.04 \\
\hline & & & 15 & 348 & 0.76 & 0.78 & \pm & 0.05 \\
\hline & & & 14 & 396 & 1.04 & 1.07 & \pm & 0.07 \\
\hline & & & 13 & 496 & 0.96 & 0.98 & \pm & 0.07 \\
\hline
\end{tabular}




\begin{tabular}{|c|c|c|c|c|c|c|c|c|}
\hline & & & 12 & 595 & 1.03 & 1.06 & \pm & 0.07 \\
\hline & & & 11 & 792 & 1.21 & 1.24 & \pm & 0.08 \\
\hline & & & 10 & 990 & 1.08 & 1.11 & \pm & 0.08 \\
\hline & & & 9 & 1188 & 1.35 & 1.38 & \pm & 0.09 \\
\hline & & & 8 & 1385 & 1.23 & 1.26 & \pm & 0.09 \\
\hline & & & 7 & 1581 & 1.31 & 1.34 & \pm & 0.09 \\
\hline & & & 4 & 2465 & 1.25 & 1.28 & \pm & 0.09 \\
\hline & & & 3 & 2955 & 1.27 & 1.30 & \pm & 0.09 \\
\hline & & & 2 & 3444 & 1.31 & 1.34 & \pm & 0.09 \\
\hline 19 & 45.05 & -18.505 & 24 & 20 & 0.09 & 0.09 & \pm & 0.01 \\
\hline & & & 23 & 30 & 0.31 & 0.32 & \pm & 0.03 \\
\hline & & & 22 & 40 & 0.17 & 0.17 & \pm & 0.02 \\
\hline & & & 21 & 50 & 0.10 & 0.10 & \pm & 0.01 \\
\hline & & & 20 & 99 & 0.17 & 0.17 & \pm & 0.02 \\
\hline & & & 19 & 200 & 0.31 & 0.32 & \pm & 0.03 \\
\hline & & & 16 & 298 & 0.23 & 0.24 & \pm & 0.02 \\
\hline & & & 15 & 397 & 0.36 & 0.37 & \pm & 0.04 \\
\hline & & & 14 & 496 & 0.48 & 0.49 & \pm & 0.05 \\
\hline & & & 13 & 595 & 0.53 & 0.54 & \pm & 0.05 \\
\hline & & & 12 & 793 & 0.71 & 0.73 & \pm & 0.07 \\
\hline & & & 11 & 991 & 0.74 & 0.76 & \pm & 0.07 \\
\hline & & & 10 & 1188 & 0.78 & 0.80 & \pm & 0.08 \\
\hline & & & 9 & 1386 & 0.68 & 0.70 & \pm & 0.07 \\
\hline & & & 8 & 1582 & 0.77 & 0.79 & \pm & 0.08 \\
\hline & & & 7 & 1779 & 0.78 & 0.80 & \pm & 0.08 \\
\hline & & & 6 & 1975 & 0.80 & 0.82 & \pm & 0.08 \\
\hline & & & 5 & 2221 & 0.86 & 0.88 & \pm & 0.09 \\
\hline & & & 4 & 2466 & 1.13 & 1.16 & \pm & 0.11 \\
\hline & & & 3 & 2955 & 0.87 & 0.89 & \pm & 0.09 \\
\hline & & & 2 & 3930 & 0.99 & 1.01 & \pm & 0.10 \\
\hline & & & 1 & 4538 & 0.99 & 1.01 & \pm & 0.10 \\
\hline 21 & 46.544 & -19.672 & 24 & 19 & 0.17 & 0.17 & \pm & 0.01 \\
\hline & & & 23 & 30 & 0.28 & 0.29 & \pm & 0.02 \\
\hline & & & 22 & 49 & 0.20 & 0.21 & \pm & 0.01 \\
\hline & & & 21 & 79 & 0.14 & 0.14 & \pm & 0.01 \\
\hline & & & 20 & 99 & 0.17 & 0.17 & \pm & 0.01 \\
\hline
\end{tabular}




\begin{tabular}{|c|c|c|c|c|c|c|c|c|}
\hline & & & 19 & 198 & 0.24 & 0.25 & \pm & 0.02 \\
\hline & & & 16 & 297 & 0.34 & 0.35 & \pm & 0.02 \\
\hline & & & 15 & 397 & 0.35 & 0.36 & \pm & 0.02 \\
\hline & & & 14 & 496 & 0.35 & 0.36 & \pm & 0.02 \\
\hline & & & 13 & 594 & 0.35 & 0.36 & \pm & 0.02 \\
\hline & & & 12 & 693 & 0.53 & 0.54 & \pm & 0.04 \\
\hline & & & 11 & 792 & 0.83 & 0.85 & \pm & 0.06 \\
\hline & & & 10 & 989 & 0.79 & 0.81 & \pm & 0.06 \\
\hline & & & 9 & 1236 & 0.76 & 0.78 & \pm & 0.05 \\
\hline & & & 8 & 1482 & 0.74 & 0.76 & \pm & 0.05 \\
\hline & & & 7 & 1976 & 0.81 & 0.83 & \pm & 0.06 \\
\hline & & & 6 & 2269 & 0.88 & 0.90 & \pm & 0.06 \\
\hline & & & 5 & 2759 & 0.70 & 0.72 & \pm & 0.05 \\
\hline & & & 4 & 2955 & 0.88 & 0.90 & \pm & 0.06 \\
\hline & & & 3 & 3442 & 0.78 & 0.80 & \pm & 0.05 \\
\hline & & & 2 & 4417 & 1.29 & 1.32 & \pm & 0.09 \\
\hline & & & 1 & 4506 & 0.71 & 0.73 & \pm & 0.05 \\
\hline 23 & 48.039 & -20.848 & 24 & 20 & 0.17 & 0.17 & \pm & 0.01 \\
\hline & & & 23 & 29 & 0.18 & 0.18 & \pm & 0.01 \\
\hline & & & 22 & 40 & 0.15 & 0.15 & \pm & 0.01 \\
\hline & & & 21 & 50 & 0.18 & 0.18 & \pm & 0.01 \\
\hline & & & 20 & 60 & 0.25 & 0.26 & \pm & 0.02 \\
\hline & & & 19 & 70 & 0.28 & 0.29 & \pm & 0.02 \\
\hline & & & 16 & 100 & 0.34 & 0.35 & \pm & 0.02 \\
\hline & & & 15 & 199 & 0.73 & 0.75 & \pm & 0.05 \\
\hline & & & 14 & 397 & 0.66 & 0.68 & \pm & 0.05 \\
\hline & & & 13 & 594 & 0.32 & 0.33 & \pm & 0.02 \\
\hline & & & 12 & 792 & 0.73 & 0.75 & \pm & 0.05 \\
\hline & & & 10 & 1187 & 0.65 & 0.67 & \pm & 0.05 \\
\hline & & & 9 & 1384 & 0.69 & 0.71 & \pm & 0.05 \\
\hline & & & 8 & 1581 & 0.75 & 0.77 & \pm & 0.05 \\
\hline & & & 7 & 1778 & 0.70 & 0.72 & \pm & 0.05 \\
\hline & & & 6 & 1974 & 0.77 & 0.79 & \pm & 0.05 \\
\hline & & & 5 & 2269 & 0.92 & 0.94 & \pm & 0.06 \\
\hline & & & 4 & 2954 & 0.79 & 0.81 & \pm & 0.06 \\
\hline & & & 3 & 3443 & 0.80 & 0.82 & \pm & 0.06 \\
\hline
\end{tabular}




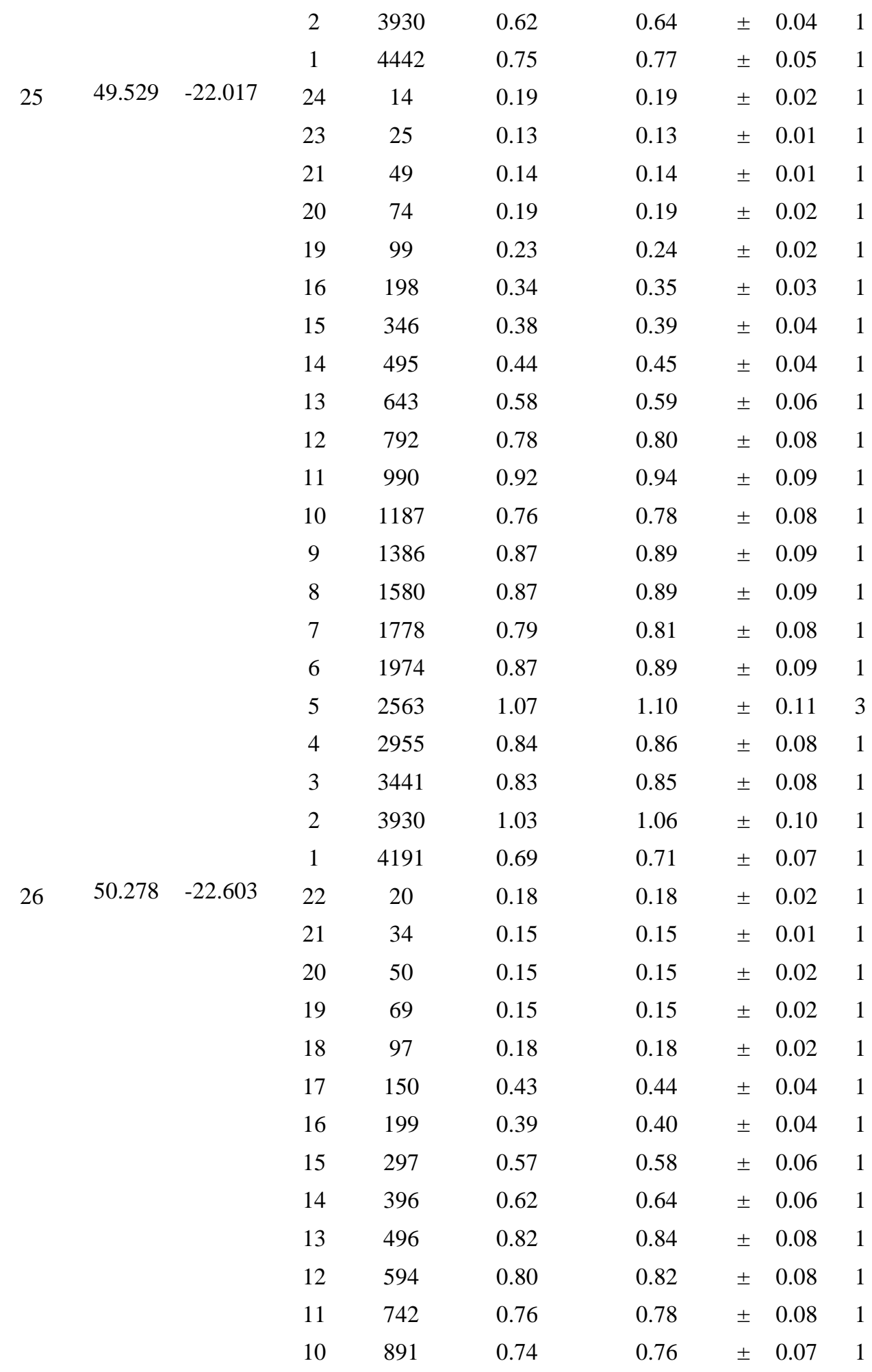




\begin{tabular}{|c|c|c|c|c|c|c|c|c|}
\hline & & & 9 & 989 & 0.83 & 0.85 & \pm & 0.08 \\
\hline & & & 8 & 1186 & 0.89 & 0.91 & \pm & 0.09 \\
\hline & & & 7 & 1384 & 0.83 & 0.85 & \pm & 0.08 \\
\hline & & & 6 & 1580 & 0.92 & 0.94 & \pm & 0.09 \\
\hline & & & 5 & 1974 & 0.90 & 0.92 & \pm & 0.09 \\
\hline & & & 4 & 2268 & 0.87 & 0.89 & \pm & 0.09 \\
\hline & & & 3 & 2953 & 0.75 & 0.77 & \pm & 0.08 \\
\hline & & & 2 & 3929 & 0.61 & 0.63 & \pm & 0.06 \\
\hline & & & 1 & 4116 & 0.63 & 0.65 & \pm & 0.06 \\
\hline 29 & 53.019 & -24.752 & 22 & 15 & 0.17 & 0.17 & \pm & 0.02 \\
\hline & & & 21 & 25 & 0.75 & 0.77 & \pm & 0.08 \\
\hline & & & 20 & 50 & 0.66 & 0.68 & \pm & 0.07 \\
\hline & & & 19 & 75 & 0.52 & 0.53 & \pm & 0.05 \\
\hline & & & 18 & 99 & 0.64 & 0.66 & \pm & 0.06 \\
\hline & & & 17 & 149 & 0.71 & 0.73 & \pm & 0.07 \\
\hline & & & 16 & 198 & 1.14 & 1.17 & \pm & 0.11 \\
\hline & & & 15 & 298 & 1.02 & 1.05 & \pm & 0.10 \\
\hline & & & 14 & 397 & 0.98 & 1.00 & \pm & 0.10 \\
\hline & & & 13 & 495 & 0.95 & 0.97 & \pm & 0.09 \\
\hline & & & 11 & 791 & 0.98 & 1.00 & \pm & 0.10 \\
\hline & & & 10 & 890 & 1.10 & 1.13 & \pm & 0.11 \\
\hline & & & 9 & 1087 & 0.99 & 1.01 & \pm & 0.10 \\
\hline & & & 8 & 1185 & 0.85 & 0.87 & \pm & 0.09 \\
\hline & & & 7 & 1382 & 0.92 & 0.94 & \pm & 0.09 \\
\hline & & & 6 & 1581 & 0.93 & 0.95 & \pm & 0.09 \\
\hline & & & 5 & 1776 & 0.71 & 0.73 & \pm & 0.07 \\
\hline & & & 4 & 1973 & 0.65 & 0.67 & \pm & 0.06 \\
\hline & & & 3 & 2464 & 1.09 & 1.12 & \pm & 0.11 \\
\hline & & & 2 & 2953 & 0.98 & 1.00 & \pm & 0.10 \\
\hline & & & 1 & 3522 & 0.69 & 0.71 & \pm & 0.07 \\
\hline 32 & 55.506 & -26.71 & 22 & 16 & 0.52 & 0.53 & \pm & 0.05 \\
\hline & & & 21 & 26 & 0.64 & 0.66 & \pm & 0.06 \\
\hline & & & 20 & 51 & 0.18 & 0.18 & \pm & 0.02 \\
\hline & & & 19 & 101 & 0.22 & 0.23 & \pm & 0.02 \\
\hline & & & 18 & 198 & 0.39 & 0.40 & \pm & 0.04 \\
\hline & & & 17 & 298 & 0.49 & 0.50 & \pm & 0.05 \\
\hline
\end{tabular}




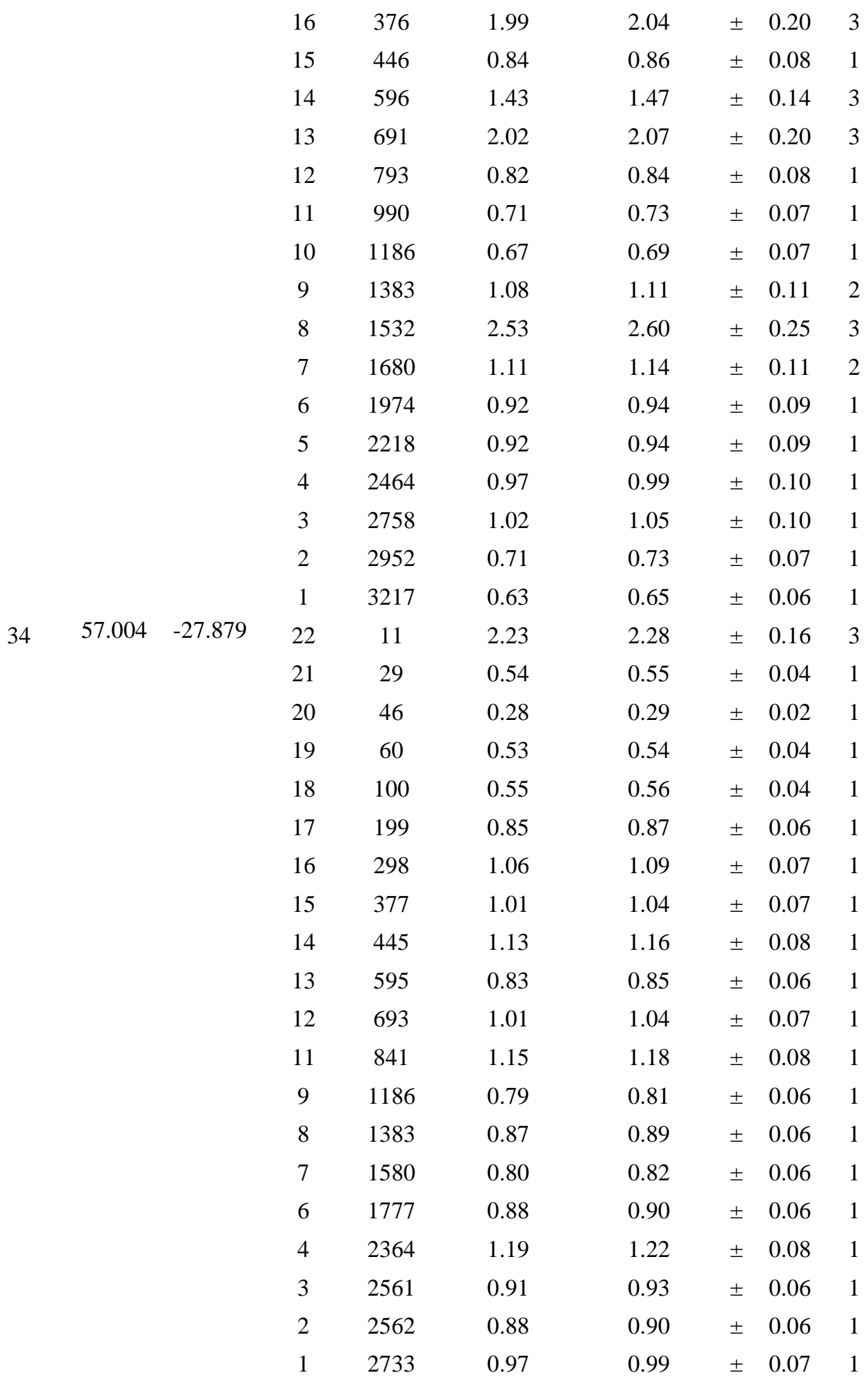




\begin{tabular}{|c|c|c|c|c|c|c|c|c|}
\hline \multirow[t]{11}{*}{36} & 58.207 & -29.725 & 20 & 20 & 0.12 & 0.12 & \pm & 0.02 \\
\hline & & & 19 & 39 & 0.09 & 0.09 & \pm & 0.01 \\
\hline & & & 18 & 69 & 0.15 & 0.15 & \pm & 0.02 \\
\hline & & & 16 & 124 & 0.19 & 0.19 & \pm & 0.03 \\
\hline & & & 14 & 247 & 0.28 & 0.29 & \pm & 0.04 \\
\hline & & & 12 & 494 & 0.69 & 0.71 & \pm & 0.10 \\
\hline & & & 10 & 839 & 1.61 & 1.65 & \pm & 0.24 \\
\hline & & & 8 & 1204 & 1.15 & 1.18 & \pm & 0.17 \\
\hline & & & 6 & 1480 & 0.89 & 0.91 & \pm & 0.13 \\
\hline & & & 4 & 1775 & 1.23 & 1.26 & \pm & 0.18 \\
\hline & & & 2 & 2212 & 1.48 & 1.52 & \pm & 0.22 \\
\hline \multirow[t]{18}{*}{38} & 58.843 & -31.267 & 21 & 20 & 0.46 & 0.47 & \pm & 0.07 \\
\hline & & & 20 & 30 & 0.24 & 0.25 & \pm & 0.04 \\
\hline & & & 19 & 49 & 0.26 & 0.27 & \pm & 0.04 \\
\hline & & & 17 & 59 & 0.31 & 0.32 & \pm & 0.05 \\
\hline & & & 16 & 69 & 0.27 & 0.28 & \pm & 0.04 \\
\hline & & & 14 & 100 & 0.37 & 0.38 & \pm & 0.06 \\
\hline & & & 12 & 199 & 0.61 & 0.63 & \pm & 0.09 \\
\hline & & & 11 & 297 & 0.62 & 0.64 & \pm & 0.09 \\
\hline & & & 10 & 397 & 0.62 & 0.64 & \pm & 0.09 \\
\hline & & & 9 & 495 & 0.93 & 0.95 & \pm & 0.14 \\
\hline & & & 8 & 569 & 0.69 & 0.71 & \pm & 0.10 \\
\hline & & & 7 & 644 & 0.78 & 0.80 & \pm & 0.12 \\
\hline & & & 6 & 792 & 0.69 & 0.71 & \pm & 0.10 \\
\hline & & & 5 & 940 & 1.12 & 1.15 & \pm & 0.17 \\
\hline & & & 4 & 990 & 1.21 & 1.24 & \pm & 0.18 \\
\hline & & & 3 & 1149 & 1.08 & 1.11 & \pm & 0.16 \\
\hline & & & 2 & 1285 & 0.74 & 0.76 & \pm & 0.11 \\
\hline & & & 1 & 1337 & 1.23 & 1.26 & \pm & 0.19 \\
\hline \multirow[t]{7}{*}{40} & 59.102 & -33.828 & 22 & 20 & 0.38 & 0.39 & \pm & 0.05 \\
\hline & & & 20 & 37 & 0.56 & 0.57 & \pm & 0.08 \\
\hline & & & 18 & 68 & 0.66 & 0.68 & \pm & 0.09 \\
\hline & & & 16 & 149 & 0.84 & 0.86 & \pm & 0.12 \\
\hline & & & 14 & 346 & 0.89 & 0.91 & \pm & 0.12 \\
\hline & & & 12 & 593 & 0.95 & 0.97 & \pm & 0.13 \\
\hline & & & 10 & 988 & 1.19 & 1.22 & \pm & 0.17 \\
\hline
\end{tabular}




\begin{tabular}{|c|c|c|c|c|c|c|c|c|}
\hline & & & 8 & 1282 & 3.30 & 3.38 & \pm & 0.46 \\
\hline & & & 6 & 1578 & 2.13 & 2.18 & \pm & 0.30 \\
\hline & & & 4 & 2069 & 1.37 & 1.40 & \pm & 0.19 \\
\hline & & & 2 & 2273 & 1.25 & 1.28 & \pm & 0.17 \\
\hline \multirow[t]{22}{*}{42} & 59.363 & -36.397 & 22 & 20 & 0.35 & 0.36 & \pm & 0.05 \\
\hline & & & 21 & 35 & 0.69 & 0.71 & \pm & 0.10 \\
\hline & & & 20 & 50 & 0.37 & 0.38 & \pm & 0.05 \\
\hline & & & 19 & 70 & 0.57 & 0.58 & \pm & 0.08 \\
\hline & & & 18 & 99 & 0.97 & 0.99 & \pm & 0.14 \\
\hline & & & 17 & 199 & 0.95 & 0.97 & \pm & 0.13 \\
\hline & & & 16 & 297 & 0.71 & 0.73 & \pm & 0.10 \\
\hline & & & 15 & 397 & 0.85 & 0.87 & \pm & 0.12 \\
\hline & & & 14 & 495 & 0.78 & 0.80 & \pm & 0.11 \\
\hline & & & 13 & 693 & 0.84 & 0.86 & \pm & 0.12 \\
\hline & & & 12 & 890 & 1.06 & 1.09 & \pm & 0.15 \\
\hline & & & 11 & 1038 & 0.82 & 0.84 & \pm & 0.11 \\
\hline & & & 10 & 1186 & 0.91 & 0.93 & \pm & 0.13 \\
\hline & & & 9 & 1383 & 1.69 & 1.73 & \pm & 0.24 \\
\hline & & & 8 & 1580 & 2.39 & 2.45 & \pm & 0.34 \\
\hline & & & 7 & 1777 & 1.09 & 1.12 & \pm & 0.15 \\
\hline & & & 6 & 1973 & 1.93 & 1.98 & \pm & 0.27 \\
\hline & & & 5 & 2217 & 1.27 & 1.30 & \pm & 0.18 \\
\hline & & & 4 & 2462 & 1.44 & 1.48 & \pm & 0.20 \\
\hline & & & 3 & 2854 & 1.02 & 1.05 & \pm & 0.14 \\
\hline & & & 2 & 3048 & 1.64 & 1.68 & \pm & 0.23 \\
\hline & & & 1 & 3078 & 0.96 & 0.98 & \pm & 0.13 \\
\hline \multirow[t]{10}{*}{44} & 59.623 & -38.954 & 22 & 21 & 1.33 & 1.37 & \pm & 0.13 \\
\hline & & & 21 & 31 & 0.54 & 0.55 & \pm & 0.05 \\
\hline & & & 20 & 40 & 0.50 & 0.51 & \pm & 0.05 \\
\hline & & & 19 & 50 & 1.01 & 1.04 & \pm & 0.10 \\
\hline & & & 18 & 100 & 1.25 & 1.28 & \pm & 0.12 \\
\hline & & & 17 & 198 & 0.80 & 0.82 & \pm & 0.08 \\
\hline & & & 16 & 299 & 0.65 & 0.67 & \pm & 0.06 \\
\hline & & & 15 & 496 & 2.08 & 2.13 & \pm & 0.21 \\
\hline & & & 14 & 693 & 0.98 & 1.00 & \pm & 0.10 \\
\hline & & & 13 & 891 & 1.23 & 1.26 & \pm & 0.12 \\
\hline
\end{tabular}




\begin{tabular}{|c|c|c|c|c|c|c|c|c|}
\hline & & & 12 & 990 & 0.73 & 0.75 & \pm & 0.07 \\
\hline & & & 11 & 1087 & 0.96 & 0.98 & \pm & 0.10 \\
\hline & & & 10 & 1236 & 1.14 & 1.17 & \pm & 0.11 \\
\hline & & & 9 & 1382 & 1.14 & 1.17 & \pm & 0.11 \\
\hline & & & 8 & 1581 & 1.25 & 1.28 & \pm & 0.12 \\
\hline & & & 7 & 1776 & 1.20 & 1.23 & \pm & 0.12 \\
\hline & & & 6 & 1972 & 1.05 & 1.08 & \pm & 0.11 \\
\hline & & & 5 & 2218 & 1.70 & 1.74 & \pm & 0.17 \\
\hline & & & 4 & 2560 & 2.45 & 2.51 & \pm & 0.25 \\
\hline & & & 3 & 2754 & 1.99 & 2.04 & \pm & 0.20 \\
\hline & & & 2 & 2854 & 1.14 & 1.17 & \pm & 0.11 \\
\hline & & & 1 & 2915 & 1.34 & 1.37 & \pm & 0.13 \\
\hline 49 & 59.773 & -41.297 & 22 & 19 & 0.25 & 0.26 & \pm & 0.02 \\
\hline & & & 21 & 40 & 0.33 & 0.34 & \pm & 0.02 \\
\hline & & & 20 & 61 & 0.42 & 0.43 & \pm & 0.03 \\
\hline & & & 19 & 80 & 0.45 & 0.46 & \pm & 0.03 \\
\hline & & & 18 & 101 & 0.45 & 0.46 & \pm & 0.03 \\
\hline & & & 17 & 198 & 0.89 & 0.91 & \pm & 0.06 \\
\hline & & & 16 & 297 & 0.96 & 0.98 & \pm & 0.07 \\
\hline & & & 15 & 396 & 1.18 & 1.21 & \pm & 0.08 \\
\hline & & & 14 & 544 & 1.75 & 1.80 & \pm & 0.12 \\
\hline & & & 13 & 593 & 1.02 & 1.05 & \pm & 0.07 \\
\hline & & & 12 & 693 & 0.98 & 1.00 & \pm & 0.07 \\
\hline & & & 11 & 792 & 1.17 & 1.20 & \pm & 0.08 \\
\hline & & & 10 & 891 & 0.97 & 0.99 & \pm & 0.07 \\
\hline & & & 9 & 989 & 1.15 & 1.18 & \pm & 0.08 \\
\hline & & & 8 & 1088 & 1.78 & 1.82 & \pm & 0.12 \\
\hline & & & 7 & 1235 & 1.25 & 1.28 & \pm & 0.09 \\
\hline & & & 6 & 1482 & 1.32 & 1.35 & \pm & 0.09 \\
\hline & & & 5 & 1629 & 1.53 & 1.57 & \pm & 0.11 \\
\hline & & & 4 & 1776 & 1.12 & 1.15 & \pm & 0.08 \\
\hline & & & 3 & 1873 & 1.35 & 1.38 & \pm & 0.09 \\
\hline & & & 2 & 1972 & 1.26 & 1.29 & \pm & 0.09 \\
\hline & & & 1 & 2022 & 1.11 & 1.14 & \pm & 0.08 \\
\hline 53 & 59.902 & -43.015 & 16 & 55 & 1.17 & 1.20 & \pm & 0.12 \\
\hline & & & 12 & 70 & 1.43 & 1.47 & \pm & 0.14 \\
\hline
\end{tabular}




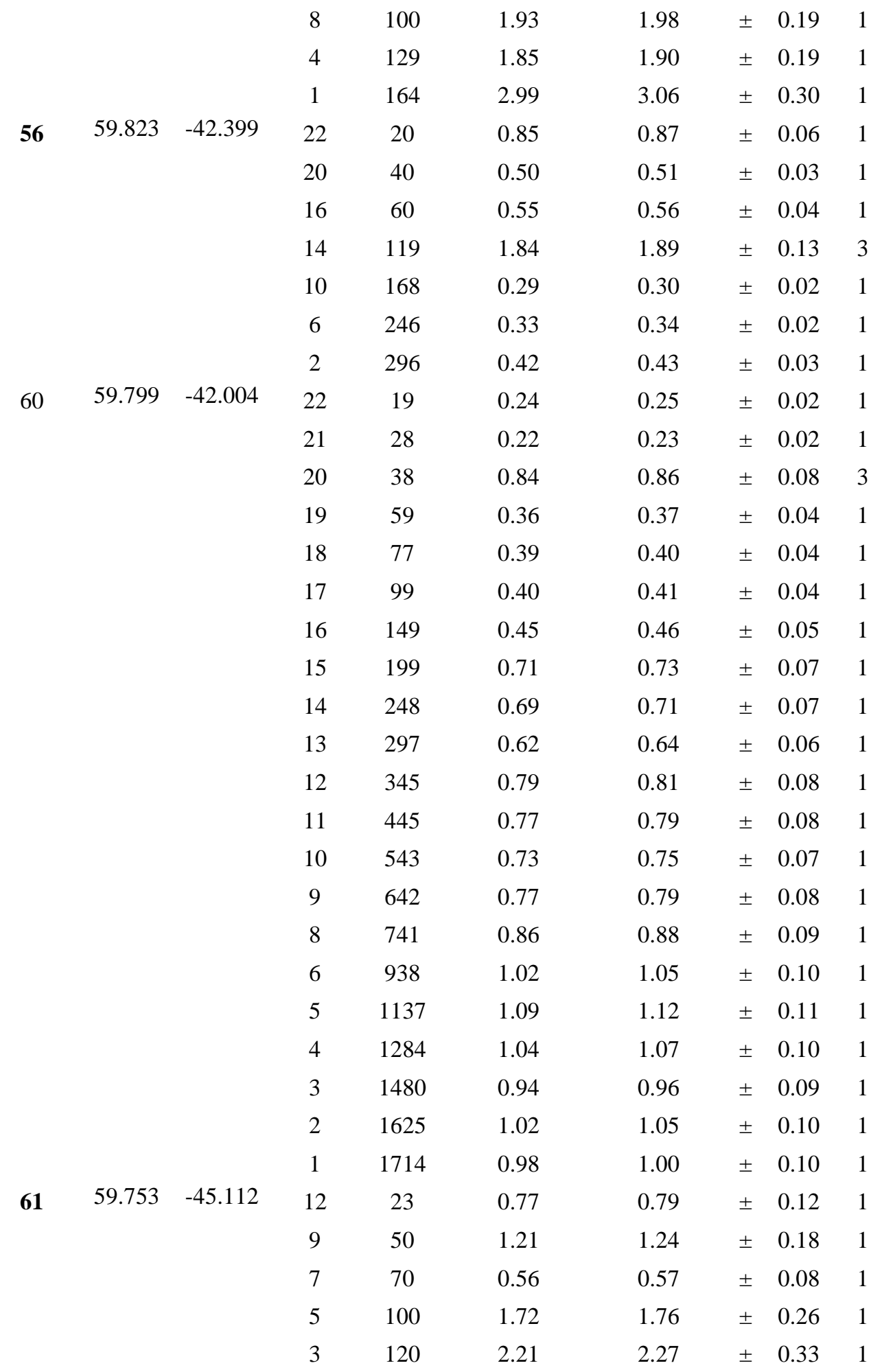




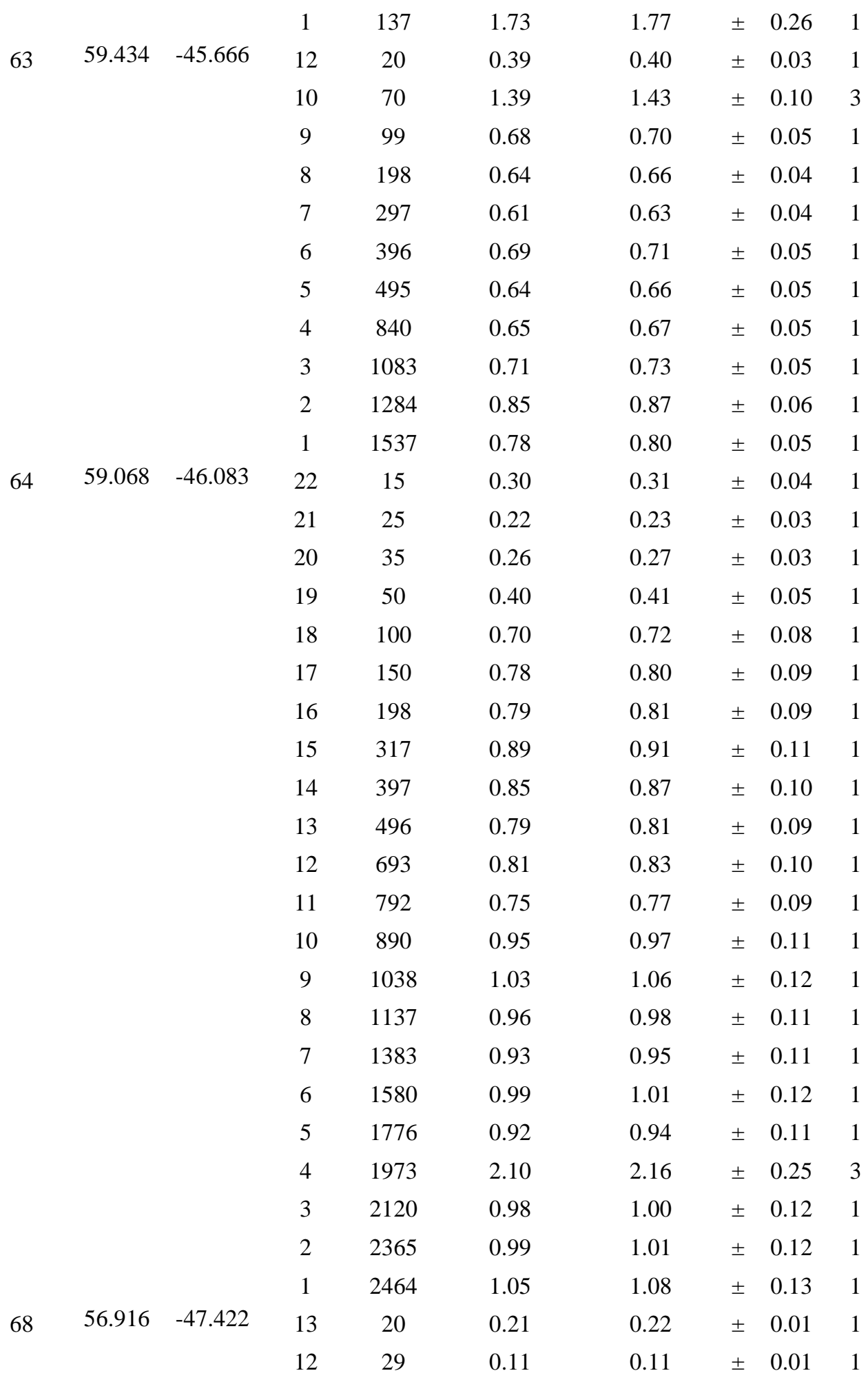




\begin{tabular}{|c|c|c|c|c|c|c|c|c|}
\hline & & & 10 & 35 & 0.26 & 0.27 & \pm & 0.02 \\
\hline & & & 9 & 50 & 0.30 & 0.31 & \pm & 0.02 \\
\hline & & & 8 & 100 & 0.73 & 0.75 & \pm & 0.05 \\
\hline & & & 7 & 345 & 0.62 & 0.64 & \pm & 0.04 \\
\hline & & & 6 & 891 & 0.69 & 0.71 & \pm & 0.05 \\
\hline & & & 5 & 1382 & 0.61 & 0.63 & \pm & 0.04 \\
\hline & & & 4 & 1677 & 0.71 & 0.73 & \pm & 0.05 \\
\hline & & & 3 & 2463 & 0.85 & 0.87 & \pm & 0.06 \\
\hline & & & 2 & 3342 & 0.68 & 0.70 & \pm & 0.05 \\
\hline & & & 1 & 3574 & 0.68 & 0.70 & \pm & 0.05 \\
\hline 69 & 55.842 & -48.093 & 22 & 14 & 0.23 & 0.24 & \pm & 0.02 \\
\hline & & & 21 & 26 & 0.18 & 0.18 & \pm & 0.01 \\
\hline & & & 20 & 30 & 0.22 & 0.23 & \pm & 0.02 \\
\hline & & & 19 & 40 & 0.35 & 0.36 & \pm & 0.02 \\
\hline & & & 18 & 60 & 0.64 & 0.65 & \pm & 0.04 \\
\hline & & & 17 & 90 & 0.47 & 0.48 & \pm & 0.03 \\
\hline & & & 16 & 128 & 0.62 & 0.64 & \pm & 0.04 \\
\hline & & & 15 & 178 & 0.64 & 0.66 & \pm & 0.04 \\
\hline & & & 14 & 495 & 0.86 & 0.88 & \pm & 0.06 \\
\hline & & & 13 & 792 & 0.74 & 0.76 & \pm & 0.05 \\
\hline & & & 12 & 1087 & 1.10 & 1.13 & \pm & 0.08 \\
\hline & & & 10 & 1381 & 0.79 & 0.81 & \pm & 0.06 \\
\hline & & & 9 & 1580 & 0.90 & 0.92 & \pm & 0.06 \\
\hline & & & 8 & 1776 & 0.84 & 0.86 & \pm & 0.06 \\
\hline & & & 7 & 2071 & 0.78 & 0.80 & \pm & 0.05 \\
\hline & & & 6 & 2365 & 0.95 & 0.97 & \pm & 0.07 \\
\hline & & & 5 & 2757 & 0.95 & 0.97 & \pm & 0.07 \\
\hline & & & 4 & 3196 & 0.76 & 0.78 & \pm & 0.05 \\
\hline & & & 3 & 3440 & 0.60 & 0.62 & \pm & 0.04 \\
\hline & & & 2 & 3635 & 0.63 & 0.65 & \pm & 0.04 \\
\hline & & & 1 & 3669 & 0.61 & 0.63 & \pm & 0.04 \\
\hline 71 & 53.692 & -49.433 & 22 & 20 & 0.28 & 0.29 & \pm & 0.02 \\
\hline & & & 21 & 30 & 0.34 & 0.35 & \pm & 0.02 \\
\hline & & & 20 & 40 & 0.53 & 0.54 & \pm & 0.04 \\
\hline & & & 19 & 50 & 0.22 & 0.23 & \pm & 0.02 \\
\hline & & & 18 & 60 & 0.33 & 0.34 & \pm & 0.02 \\
\hline
\end{tabular}




\begin{tabular}{|c|c|c|c|c|c|c|c|c|}
\hline & & & 17 & 100 & 0.61 & 0.63 & \pm & 0.04 \\
\hline & & & 16 & 149 & 0.64 & 0.66 & \pm & 0.04 \\
\hline & & & 15 & 248 & 0.73 & 0.75 & \pm & 0.05 \\
\hline & & & 14 & 347 & 0.79 & 0.81 & \pm & 0.05 \\
\hline & & & 13 & 496 & 0.81 & 0.83 & \pm & 0.06 \\
\hline & & & 12 & 792 & 0.67 & 0.69 & \pm & 0.05 \\
\hline & & & 10 & 1187 & 0.81 & 0.83 & \pm & 0.06 \\
\hline & & & 9 & 1383 & 0.84 & 0.86 & \pm & 0.06 \\
\hline & & & 8 & 1678 & 2.17 & 2.23 & \pm & 0.15 \\
\hline & & & 7 & 1974 & 0.72 & 0.74 & \pm & 0.05 \\
\hline & & & 6 & 2366 & 0.78 & 0.80 & \pm & 0.05 \\
\hline & & & 5 & 2709 & 1.02 & 1.04 & \pm & 0.07 \\
\hline & & & 4 & 2952 & 0.83 & 0.85 & \pm & 0.06 \\
\hline & & & 2 & 3440 & 0.68 & 0.70 & \pm & 0.05 \\
\hline & & & 1 & 3689 & 0.73 & 0.75 & \pm & 0.05 \\
\hline 77 & 53 & -51.1 & 22 & 15 & 0.28 & 0.29 & \pm & 0.02 \\
\hline & & & 21 & 30 & 0.26 & 0.27 & \pm & 0.02 \\
\hline & & & 20 & 40 & 0.31 & 0.32 & \pm & 0.02 \\
\hline & & & 19 & 60 & 0.55 & 0.56 & \pm & 0.04 \\
\hline & & & 18 & 80 & 0.56 & 0.57 & \pm & 0.04 \\
\hline & & & 17 & 100 & 0.77 & 0.79 & \pm & 0.05 \\
\hline & & & 16 & 149 & 0.90 & 0.92 & \pm & 0.06 \\
\hline & & & 15 & 396 & 1.21 & 1.24 & \pm & 0.09 \\
\hline & & & 14 & 693 & 0.96 & 0.98 & \pm & 0.07 \\
\hline & & & 13 & 989 & 0.83 & 0.85 & \pm & 0.06 \\
\hline & & & 12 & 1088 & 0.87 & 0.89 & \pm & 0.06 \\
\hline & & & 10 & 1186 & 0.96 & 0.98 & \pm & 0.07 \\
\hline & & & 9 & 1285 & 0.95 & 0.97 & \pm & 0.07 \\
\hline & & & 8 & 1482 & 0.90 & 0.92 & \pm & 0.06 \\
\hline & & & 7 & 1679 & 0.95 & 0.97 & \pm & 0.07 \\
\hline & & & 6 & 1875 & 0.92 & 0.94 & \pm & 0.06 \\
\hline & & & 5 & 2072 & 1.01 & 1.04 & \pm & 0.07 \\
\hline & & & 4 & 2366 & 0.94 & 0.96 & \pm & 0.07 \\
\hline & & & 2 & 2415 & 0.93 & 0.95 & \pm & 0.07 \\
\hline & & & 1 & 2506 & 0.88 & 0.90 & \pm & 0.06 \\
\hline 78 & 51.989 & -53.817 & 12 & 12 & 0.77 & 0.79 & \pm & 0.05 \\
\hline
\end{tabular}




\begin{tabular}{ccccccc}
9 & 36 & 1.15 & 1.18 & \pm & 0.08 & 1 \\
7 & 139 & 2.36 & 2.42 & \pm & 0.17 & 1 \\
5 & 248 & 4.02 & 4.12 & \pm & 0.28 & 1 \\
4 & 288 & 3.23 & 3.31 & \pm & 0.23 & 1 \\
1 & 368 & 7.64 & 7.83 & \pm & 0.53 & 1 \\
\hline
\end{tabular}




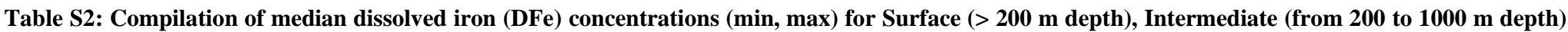

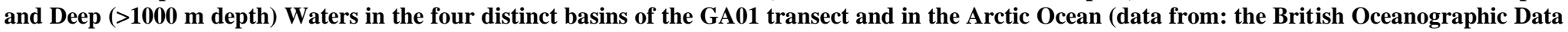

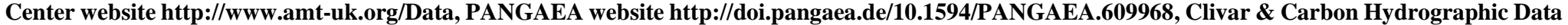

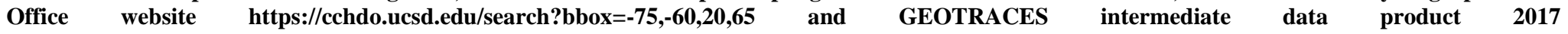

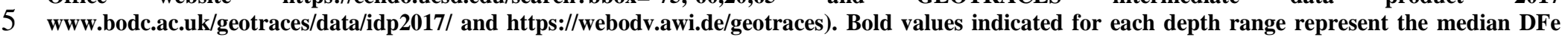
concentrations all studies considered per basin.

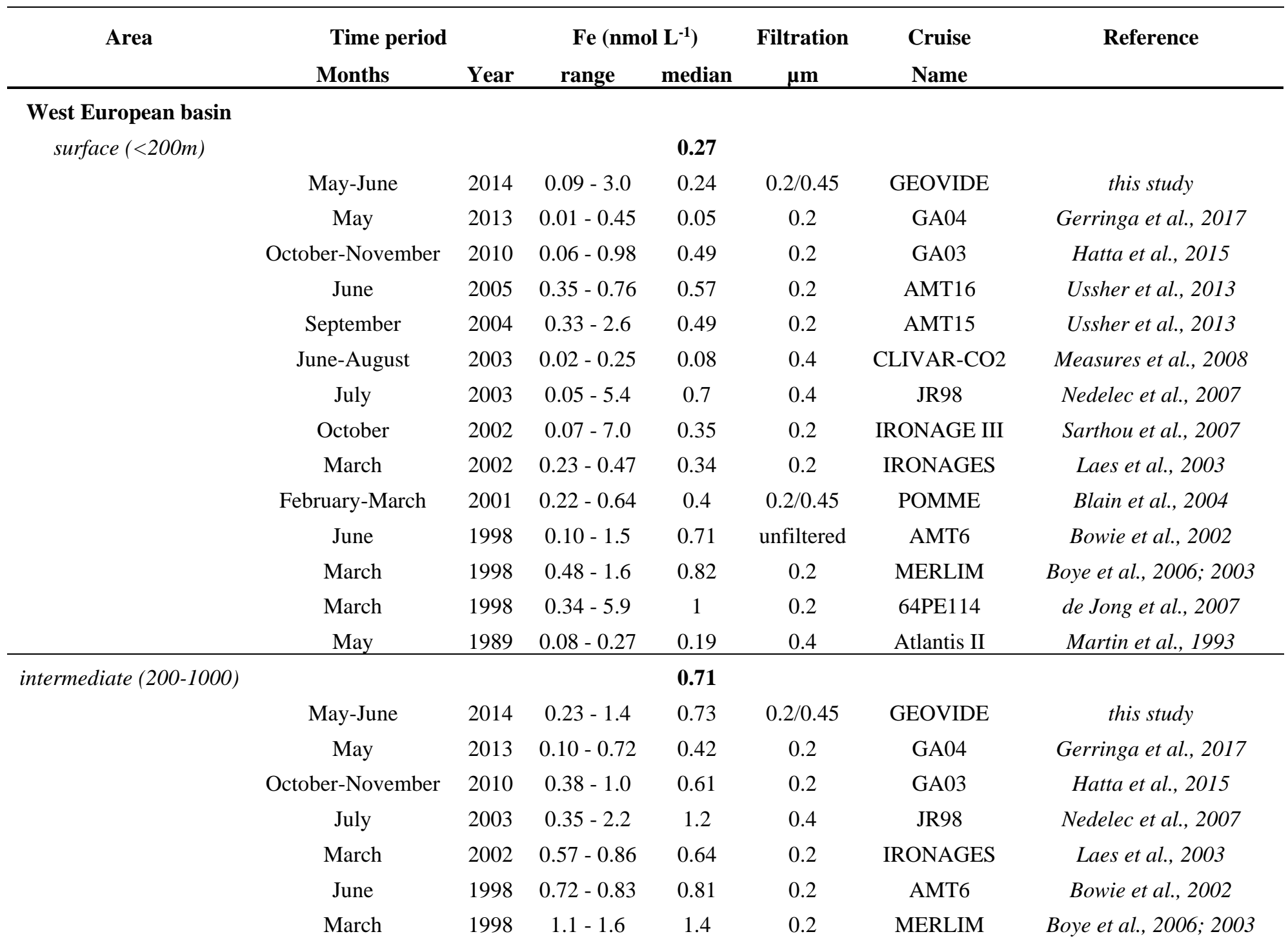




\begin{tabular}{|c|c|c|c|c|c|c|c|}
\hline & March & 1998 & $1.3-1.9$ & 1.6 & 0.2 & 64PE114 & de Jong et al., 2007 \\
\hline & May & 1989 & $0.26-0.57$ & 0.35 & 0.4 & Atlantis II & Martin et al., 1993 \\
\hline \multirow[t]{7}{*}{$\operatorname{deep}(>1000)$} & & & & 0.76 & & & \\
\hline & May & 2013 & $0.44-0.87$ & 0.61 & 0.2 & GA04 & Gerringa et al., 2017 \\
\hline & October-November & 2010 & $0.46-1.1$ & 0.75 & 0.2 & GA03 & Hatta et al., 2015 \\
\hline & June & 1998 & $0.57-0.94$ & 0.76 & 0.2 & AMT6 & Bowie et al., 2002 \\
\hline & March & 1998 & $1.3-1.6$ & 1.4 & 0.2 & MERLIM & Boye et al., 2006; 2003 \\
\hline & March & 1998 & $1.3-2.0$ & 1.7 & 0.2 & 64PE114 & de Jong et al., 2007 \\
\hline & May & 1989 & $0.54-0.66$ & 0.6 & 0.4 & Atlantis II & Martin et al., 1993 \\
\hline \multicolumn{8}{|l|}{ Iceland Basin } \\
\hline \multirow{7}{*}{ surface $(<200 m)$} & July-August & 2010 & $0.03-2.6$ & 0.25 & 0.2 & D354 & Achterberg et al., 2018 \\
\hline & April-May & 2010 & $0.11-2.6$ & 0.3 & 0.2 & D350 & Achterberg et al., 2018 \\
\hline & June & 2009 & $0.08-0.87$ & 0.24 & 0.2 & D340 & Mohamed et al., 2011 \\
\hline & August-September & 2007 & $0.04-0.34$ & 0.14 & 0.2 & D321 & Mohamed et al., 2011 \\
\hline & July-September & 2007 & $0.02-0.41$ & 0.06 & 0.2 & & Nielsdottir et al., 2009 \\
\hline & June-August & 2003 & $0.02-0.30$ & 0.1 & 0.4 & CLIVAR-CO2 & Measures et al., 2008 \\
\hline & May & 1989 & $0.06-0.23$ & 0.12 & 0.4 & Atlantis II & Martin et al., 1993 \\
\hline \multirow[t]{3}{*}{ intermediate (200-1000) } & & & & 0.71 & & & \\
\hline & May-June & 2014 & $0.28-1.6$ & 0.94 & $0.2 / 0.45$ & GEOVIDE & this study \\
\hline & May & 1989 & $0.17-0.54$ & 0.37 & 0.4 & Atlantis II & Martin et al., 1993 \\
\hline
\end{tabular}




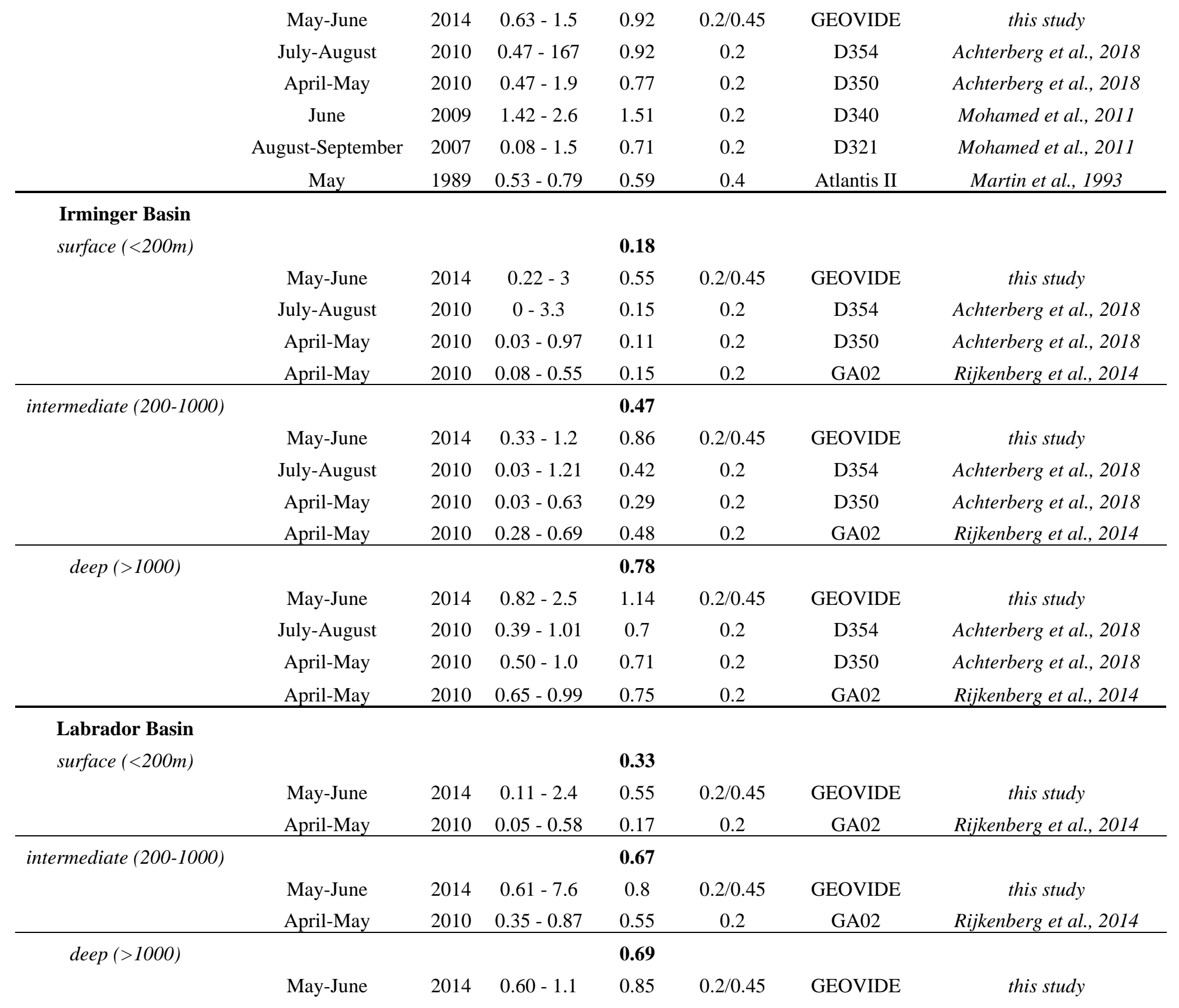


April-May

$2010 \quad 0.47-0.66$

0.59

0.2

GA02

Rijkenberg et al., 2014

Arctic Ocean

surface $(<200 m)$

$\begin{array}{ccc}\text { July } & 2008 & 2.1-16 \\ \text { September } & 2008 & 0.5-3.2 \\ \text { st - September } & 2007 & 0.10->10 \\ \text { July } & 2007 & 5.7-23\end{array}$

0.6

0.22

0.22

0.2

unfiltered

MR 08-04

Nishimura et al., 2012

August - September

$2007 \quad 0.8-3.1$

$\begin{array}{ll}1.5 & 0.4 \\ 0.5 & 0.2\end{array}$

ARK XXII/2

Nakayama et al., 2011

April-May

$\begin{array}{lll}2007 \quad 0.20-1.4 & 0.5\end{array}$

0.2

ATOS-Arctic Tovar-Sanchez et al., 2010

intermediate (200-1000)

$0.2 \quad$ ARK XXII/2
Aguilar-Islas et al., 2008

Klunder et al., 2012

Klunder et al., 2012


